\title{
A New Approach to Function Spaces on Quasi-Metric Spaces
}

\author{
Hans TRIEBEL \\ Mathematisches Institut \\ Fakultät für Mathematik und Informatik \\ Friedrich-Schiller-Universität Jena \\ D-07740 Jena, Germany \\ triebel@minet.uni-jena.de
}

Recibido: 16 de Noviembre de 2004

Aceptado: 20 de Diciembre de 2004

\begin{abstract}
A $d$-space $X=(X, \varrho, \mu)$ is a compact set $X$ with respect to a quasi-metric $\varrho$ and a Borel measure $\mu$ such that the measure of a ball of radius $r$ is equivalent to $r^{d}$, where $d>0$. The paper deals with spaces $B_{p}^{s}(X ; H)$ of Besov type where $1<p<\infty$ and $s \in \mathbb{R}$. Here $H$ is a bi-Lipschitzian map of the snowflaked version $\left(X, \varrho^{\varepsilon}, \mu\right)$ of $X$ for some $0<\varepsilon<1$, onto a fractal $d / \varepsilon$-set $\Gamma=H X$ in some $\mathbb{R}^{n}$, reducing the spaces $B_{p}^{s}(X ; H)$ to the better known spaces $B_{p}^{s / \varepsilon}(\Gamma)$.
\end{abstract}

Key words: quasi-metric spaces, snowflaked transform, Besov spaces, atomic and subatomic decompositions, entropy numbers, Riesz potentials.

2000 Mathematics Subject Classification: 46E35, 28A80, 47B06, 43A85 


\section{Contents}

$\begin{array}{ll}\text { 1. Introduction } & 9\end{array}$

2. Function spaces on $d$-sets 10

2.1. Preliminaries ..................... . . . 10

2.2. Quarkonial characterizations ................. 14

2.3. Atomic characterizations . . . . . . . . . . . . . 17

3. Quasi-metric spaces $\quad 24$

3.1. Basic assertions . . . . . . . . . . . . . . . . . . . 24

3.2. Euclidean charts . . . . . . . . . . . . . . . . . . . . . . . . . . . . . . . . . 27

3.3. Snowflaked maps . . . . . . . . . . . . . . . . . . . . 28

4. Function spaces on $d$-spaces

4.1. Frames . . . . . . . . . . . . . . . . . . . . 31

4.2. Spaces of positive smoothness . . . . . . . . . . . . . . . . . . . . . . . . . . . . . . 34

4.3. Spaces of arbitrary smoothness . . . . . . . . . . . . . . 36

4.4. The standard approach . . . . . . . . . . . . . . . . . . . . . . . . . . . . . . . . . . . . . .

4.5. Spaces of restricted smoothness . . . . . . . . . . . . . . . . . 41

5. Applications 43

5.1. Entropy numbers . . . . . . . . . . . . . . . . . . . . . . . . . . . . . . . 43

5.2. Riesz potentials . . . . . . . . . . . . . . . . . . 44

$\begin{array}{ll}\text { References } & 46\end{array}$ 


\section{Introduction}

Let $d>0$. A $d$-space $(X, \varrho, \mu)$ is a set $X$, equipped with a quasi-metric $\varrho$ and a Borel measure $\mu$ such that $(X, \varrho)$ is complete and

$$
\mu(B(x, r)) \sim r^{d}, \quad x \in X, \quad 0<r \leq \operatorname{diam} X<\infty,
$$

where $B(x, r)$ is a ball centered at $x \in X$ and of radius $r$. A $d$-set $\left(\Gamma, \varrho_{n}, \mathcal{H}_{\Gamma}^{d}\right)$ is a compact set $\Gamma$ in some $\mathbb{R}^{n}$, with the adapted counterpart of (1), where $\mu=\mathcal{H}_{\Gamma}^{d}=\mathcal{H}^{d} \mid \Gamma$ is the restriction of the Hausdorff measure $\mathcal{H}^{d}$ in $\mathbb{R}^{n}$ to $\Gamma$, and $\varrho_{n}$ is the usual Euclidean metric in $\mathbb{R}^{n}$. Obviously, $d$-sets are special $d$-spaces. But throughout the paper we shall carefully distinguish notationally between (abstract) $d$-spaces and (concrete) $d$-sets. For any $d$-space there is a number $\varepsilon_{0}$ with $0<\varepsilon_{0} \leq 1$ such that for $\varepsilon$ with $0<\varepsilon<\varepsilon_{0}$ there is a bi-Lipschitzian map

$$
H:\left(X, \varrho^{\varepsilon}, \mu\right) \Longleftrightarrow\left(\Gamma, \varrho_{n}, \mathcal{H}_{\Gamma}^{d_{\varepsilon}}\right)
$$

of the snowflaked version $\left(X, \varrho^{\varepsilon}, \mu\right)$ of $(X, \varrho, \mu)$ onto a $d_{\varepsilon}$-set $\Gamma$ in some $\mathbb{R}^{n}$ where $d_{\varepsilon}=d / \varepsilon$. We call the right-hand side of (2) an Euclidean chart or an $\varepsilon$-chart of $(X, \varrho, \mu)$ in analogy to Riemannian charts of manifolds. Let $B_{p q}^{s}\left(\mathbb{R}^{n}\right)$ be the usual Besov spaces on $\mathbb{R}^{n}$ and let $\Gamma$ be a $d$-set in $\mathbb{R}^{n}$ with $0<d<n$. For $1<p<\infty$, $0<q \leq \infty, s>0$, the trace spaces

$$
B_{p q}^{s}\left(\Gamma, \varrho_{n}, \mathcal{H}_{\Gamma}^{d}\right)=\operatorname{tr}_{\Gamma} B_{p q}^{s+\frac{n-d}{p}}\left(\mathbb{R}^{n}\right)
$$

are well defined. They can be intrinsically characterized by elementary building blocks on $\Gamma$, called $\beta$-quarks. Then the Euclidean charts according to (2) generate scales of Besov spaces

$$
B_{p}^{s}(X, \varrho, \mu ; H)=B_{p}^{s / \varepsilon}\left(\Gamma, \varrho_{n}, \mathcal{H}_{\Gamma}^{d_{\varepsilon}}\right) \circ H, \quad 1<p<\infty, \quad s>0,
$$

with $B_{p}^{\sigma}=B_{p p}^{\sigma}$. This can be extended by duality to $s<0$ and by interpolation to $s=0$. In general these spaces depend on the chosen Euclidean chart, but

$$
B_{p}^{s}(X, \varrho, \mu ; H)=B_{p}^{s}(X), \quad|s|<\varepsilon_{0}, \quad 1<p<\infty,
$$


where $\varepsilon_{0}$ has the above meaning, coincides with the Besov space $B_{p}^{s}(X)$ on the space $X$ of homogeneous type considered in the literature (references will be given later on). In particular, they are independent of the Euclidean charts.

One additional word seems to be in order. We wish to present a new method avoiding any complication which may be caused by more general function spaces or by more general underlying sets $\Gamma$ in $\mathbb{R}^{n}$ or abstract quasi-metric spaces. This might explain our restriction to the special Besov spaces $B_{p q}^{s}$ with $1<q=p<\infty$ and to $d$-spaces according to (1). There remains a substantial amount of unavoidable (?) technicalities. In other words, we follow Einstein's advice:

One should present things as simple as possible, but not simpler.

It would be of interest to extend this theory in the indicated directions. But nothing has been done so far.

In section 2 we adapt what is known so far about Besov spaces on $d$-sets in $\mathbb{R}^{n}$ to our later needs and prove some new assertions, especially about intrinsic atomic characterizations. Section 3 deals with snowflaked transforms as in (2), illustrated by some examples. In section 4 we introduce the spaces according to (3), characterizing them intrinsically by quarkonial and (if $0<s<\varepsilon_{0}$ ) by atomic decompositions, and prove (4). In section 5 we describe some applications to entropy numbers and Riesz potentials.

\section{Function spaces on $d$-sets}

\subsection{Preliminaries}

We use standard notation which will be explained when needed. In particular, let $\mathbb{R}^{n}$ be Euclidean $n$-space. Let $S\left(\mathbb{R}^{n}\right)$ and $S^{\prime}\left(\mathbb{R}^{n}\right)$ be the Schwartz space of all complexvalued rapidly decreasing $C^{\infty}$ functions on $\mathbb{R}^{n}$, and the dual space of tempered distributions. The Fourier transform of $\varphi \in S\left(\mathbb{R}^{n}\right)$ is denoted by $\widehat{\varphi}$ or $F \varphi$. As usual, $\varphi^{\vee}$ and $F^{-1} \varphi$ stand for the inverse Fourier transform. Both $F$ and $F^{-1}$ are extended to $S^{\prime}\left(\mathbb{R}^{n}\right)$ in the standard way. Let $\varphi_{0} \in S\left(\mathbb{R}^{n}\right)$ with

$$
\varphi_{0}(x)=1 \text { if }|x| \leq 1 \quad \text { and } \quad \varphi_{0}(y)=0 \text { if }|y| \geq 3 / 2
$$

and let

$$
\varphi_{k}(x)=\varphi_{0}\left(2^{-k} x\right)-\varphi_{0}\left(2^{-k+1} x\right), \quad x \in \mathbb{R}^{n}, \quad k \in \mathbb{N},
$$

where $\mathbb{N}$ is the collection of all natural numbers. Then, since $1=\sum_{j=0}^{\infty} \varphi_{j}(x)$ for all $x \in \mathbb{R}^{n}$, the $\varphi_{j}$ form a dyadic resolution of unity in $\mathbb{R}^{n}$. Recall that $\left(\varphi_{j} \widehat{f}\right)^{\vee}(x)$ makes sense pointwise since $\left(\varphi_{j} \widehat{f}\right)^{\vee}$ is an entire analytic function on $\mathbb{R}^{n}$ for any $f \in S^{\prime}\left(\mathbb{R}^{n}\right)$. 
Definition 2.1. Let $\varphi=\left\{\varphi_{j}\right\}_{j=0}^{\infty}$ be the above resolution of unity. Let $0<p \leq \infty$, $0<q \leq \infty, s \in \mathbb{R}$ and

$$
\left\|f \mid B_{p q}^{s}\left(\mathbb{R}^{n}\right)\right\|_{\varphi}=\left(\sum_{j=0}^{\infty} 2^{j s q}\left\|\left(\varphi_{j} \widehat{f}\right)^{\vee} \mid L_{p}\left(\mathbb{R}^{n}\right)\right\|^{q}\right)^{1 / q}
$$

(with the usual modification if $q=\infty$ ). Then

$$
B_{p q}^{s}\left(\mathbb{R}^{n}\right)=\left\{f \in S^{\prime}\left(\mathbb{R}^{n}\right):\left\|f \mid B_{p q}^{s}\left(\mathbb{R}^{n}\right)\right\|_{\varphi}<\infty\right\} .
$$

Remark 2.2. We assume that the reader is familiar with this Fourier-analytical approach to the spaces $B_{p q}^{s}\left(\mathbb{R}^{n}\right)$. In particular, $B_{p q}^{s}\left(\mathbb{R}^{n}\right)$ is independent of $\varphi$ (equivalent quasi-norms). They are quasi-Banach spaces (Banach spaces if $p \geq 1, q \geq 1$ ). Of course the Lebesgue spaces $L_{p}\left(\mathbb{R}^{n}\right)$, quasi-normed by

$$
\left\|f \mid L_{p}\left(\mathbb{R}^{n}\right)\right\|=\left(\int_{\mathbb{R}^{n}}|f(x)|^{p} \mathrm{~d} x\right)^{1 / p},
$$

(with the usual modification if $p=\infty$ ), have the standard meaning. We try to avoid any technical complication and restrict ourselves mostly to

$$
B_{p}^{s}\left(\mathbb{R}^{n}\right)=B_{p p}^{s}\left(\mathbb{R}^{n}\right), \quad 1<p<\infty, \quad s>0 .
$$

These are special cases of the classical Besov spaces. Otherwise a systematic study of all these spaces has been given in [39,40] and more recently in [41,42]. There one finds also the history of these spaces and many references.

Definition 2.3. Let $0<d<n \in \mathbb{N}$. A compact set $\Gamma$ in $\mathbb{R}^{n}$ is called a $d$-set if there is a Radon measure $\mu$ in $\mathbb{R}^{n}$ with $\operatorname{supp} \mu=\Gamma$ and

$$
\mu(B(\gamma, r)) \sim r^{d}, \quad \gamma \in \Gamma, \quad 0<r \leq \operatorname{diam} \Gamma
$$

where $B(\gamma, r)$ is a ball (in $\left.\mathbb{R}^{n}\right)$ centered at $\gamma$ and of radius $r$.

Remark 2.4. We use $\sim$ in this paper for two positive functions $a(w)$ and $b(w)$ if there are two positive numbers $c$ and $C$ such that

$$
c a(w) \leq b(w) \leq C a(w)
$$

for all admitted variables $w$ (discrete or continuous). If there is a Radon measure $\mu$ with the above properties then it is equivalent to $\mathcal{H}_{\Gamma}^{d}=\mathcal{H}^{d} \mid \Gamma$, the restriction of the Hausdorff measure $\mathcal{H}^{d}$ in $\mathbb{R}^{n}$ to $\Gamma$. This justifies to put $\mu=\mathcal{H}_{\Gamma}^{d}$. A short proof of this well-known fact may be found in [41, p. 5]. There are also further references especially to the standard books of fractal geometry $[12,33]$. 
Next we need traces of spaces $B_{p q}^{s}\left(\mathbb{R}^{n}\right)$ on $d$-sets $\Gamma$ in $\mathbb{R}^{n}$. Let

$$
1<p<\infty, \quad 0<q<\infty \text { and } s>0 .
$$

First one asks whether there is a constant $c>0$ such that

$$
\left\|\operatorname{tr}_{\Gamma} \varphi\left|L_{1}(\Gamma)\|\leq c\| \varphi\right| B_{p q}^{s}\left(\mathbb{R}^{n}\right)\right\|
$$

for all $\varphi \in S\left(\mathbb{R}^{n}\right)$. Obviously, $L_{r}(\Gamma)=L_{r}(\Gamma, \mu)$ with $1 \leq r<\infty$ has the usual meaning normed by

$$
\left\|f \mid L_{r}(\Gamma)\right\|=\left(\int_{\mathbb{R}^{n}}|f(x)|^{r} \mu(\mathrm{d} x)\right)^{1 / r}=\left(\int_{\Gamma}|f(\gamma)|^{r} \mu(\mathrm{d} \gamma)\right)^{1 / r} .
$$

Furthermore, $\left(\operatorname{tr}_{\Gamma} \varphi\right)(\gamma)=\varphi(\gamma)$ is the pointwise trace of $\varphi \in S\left(\mathbb{R}^{n}\right)$ on $\Gamma$. If one has (8) then one defines the trace and the trace operator $\operatorname{tr}_{\Gamma}$ by completion of $S\left(\mathbb{R}^{n}\right)$ in $B_{p q}^{s}\left(\mathbb{R}^{n}\right)$ (recall that $S\left(\mathbb{R}^{n}\right)$ is dense in $B_{p q}^{s}\left(\mathbb{R}^{n}\right)$ with $(7)$ ) and puts

$$
\operatorname{tr}_{\Gamma} B_{p q}^{s}\left(\mathbb{R}^{n}\right)=\left\{g \in L_{1}(\Gamma): g=\operatorname{tr}_{\Gamma} f \text { for some } f \in B_{p q}^{s}\left(\mathbb{R}^{n}\right)\right\}
$$

quasi-normed by

$$
\left\|g\left|\operatorname{tr}_{\Gamma} B_{p q}^{s}\left(\mathbb{R}^{n}\right)\|=\inf \| f\right| B_{p q}^{s}\left(\mathbb{R}^{n}\right)\right\|
$$

where the infimum is taken over all $f \in B_{p q}^{s}\left(\mathbb{R}^{n}\right)$ with $\operatorname{tr}_{\Gamma} f=g$. We followed essentially [42, section 9.32 , p. 151], where we dealt with more general Radon measures. In the special case of $d$-sets, hence $\mu=\mathcal{H}_{\Gamma}^{d}$, one can replace $L_{1}(\Gamma)$ on the left-hand side of $(8)$ by $L_{p}(\Gamma)$ as we did in [41, section 18.5, pp. 138-139]. The outcome is the same, [42, section $9.34(\mathrm{i})$, p. 153].

Proposition 2.5. Let $\Gamma$ be a compact d-set in $\mathbb{R}^{n}$ with $0<d<n$. Let $1<p<\infty$ and $0<q \leq 1$. Then

$$
\operatorname{tr}_{\Gamma} B_{p q}^{\frac{n-d}{p}}\left(\mathbb{R}^{n}\right)=L_{p}(\Gamma)
$$

Remark 2.6. We refer to [41, Theorem 18.6, p. 139], and the above comments. This remarkable assertion can even be extended to $0<p<\infty, 0<q \leq \min (1, p)$. This may be found in [41, Corollary 18.12, p. 142], complemented by Remark 9.19 in $[42$, p. 140]. But for our purposes the restriction to $1<p<\infty$ is sufficient. It justifies the following definition where we restrict ourselves to the case $q=p$, hence to the spaces in (5).

Definition 2.7. Let $1<p<\infty$ and $s>0$. Let $\Gamma$ be a compact $d$-set in $\mathbb{R}^{n}$ with $0<d<n$. Then

$$
B_{p}^{s}(\Gamma)=\operatorname{tr}_{\Gamma} B_{p}^{s+\frac{n-d}{p}}\left(\mathbb{R}^{n}\right)
$$

according to (9), (10). 
Remark 2.8. Proposition 2.5 justifies this definition. Otherwise (12) can be extended to $0<p \leq \infty, 0<q \leq \infty$, after an appropriate generalisation of $\operatorname{tr}_{\Gamma}$ according to (8). We refer to [41, Definition 20.2, p. 159]. Furthermore we dealt in [42], especially section 9 , with traces of spaces $B_{p q}^{s}\left(\mathbb{R}^{n}\right)$ and $F_{p q}^{s}\left(\mathbb{R}^{n}\right)$ on compact sets $\Gamma=\operatorname{supp} \mu$ for more general finite Radon measure on $\mathbb{R}^{n}$. Traces of function spaces on sets $\Gamma$ in $\mathbb{R}^{n}$ have a long history. There are the classical assertions when $\Gamma$ is a hyper-plane or the boundary of (bounded smooth) domains in $\mathbb{R}^{n}$. The history of this subject, results and the corresponding literature may be found in [39,40]. Also the case of more general (compact) sets $\Gamma$ in $\mathbb{R}^{n}$ attracted a lot of attention. One may consult the above-mentioned parts in $[41,42]$ and the references given there. We mention in particular the corresponding books $[1,34]$. The first systematic study of traces of classical Besov spaces $B_{p q}^{s}\left(\mathbb{R}^{n}\right)$ and (fractional) Sobolev spaces $H_{p}^{s}\left(\mathbb{R}^{n}\right)$ on $d$-sets in $\mathbb{R}^{n}$ is due to [27]. The respective trace spaces are characterized in terms of differences and via approximation procedures. Of interest for us is the existence of a linear extension operator. We give a corresponding formulation. Let $\mathbb{N}_{0}=\mathbb{N} \cup\{0\}$ be the collection of all non-negative integers.

Proposition 2.9. Let $\Gamma$ be a compact $d$-set in $\mathbb{R}^{n}$ with $0<d<n$. Let $1<p<\infty$, $K \in \mathbb{N}_{0}$, and $K<s<K+1$. Then there exists a common linear and bounded extension operator $\operatorname{ext}_{\Gamma}^{K}$ with

$$
\operatorname{ext}_{\Gamma}^{K}: B_{p}^{s}(\Gamma) \hookrightarrow B_{p}^{s+\frac{n-d}{p}}\left(\mathbb{R}^{n}\right)
$$

and

$$
\left.\operatorname{tr}_{\Gamma} \circ \operatorname{ext}_{\Gamma}^{K}=\mathrm{id} \quad \text { (identity in } B_{p}^{s}(\Gamma)\right)
$$

Remark 2.10. This assertion is covered by [27, Theorem 3, p. 155]. The restriction to $0<s \notin \mathbb{N}$ in $[27]$ is somewhat curious and depends on the method used there. Under some severe restrictions for the $d$-sets (satisfying the so-called Markov inequality), or if $s$ is large, then there exists an extension operator. We refer to [25,26,47]. If $p=2$ and $s>0$ then there is always a linear and bounded extension operator $\operatorname{ext}_{\Gamma, s}$,

$$
\operatorname{ext}_{\Gamma, s}: B_{2}^{s}(\Gamma) \hookrightarrow B_{2}^{s+\frac{n-d}{2}}\left(\mathbb{R}^{n}\right)
$$

such that

$$
\left.\operatorname{tr}_{\Gamma} \circ \operatorname{ext}_{\Gamma, s}=\mathrm{id} \quad \text { (identity in } B_{2}^{s}(\Gamma)\right) \text {. }
$$

But this has nothing to do with the above specific situation. In this case all spaces are Hilbert spaces and the above assertion follows from the orthogonal Weyl decomposition

$$
B_{2}^{s+\frac{n-d}{2}}\left(\mathbb{R}^{n}\right)=\left\{f \in B_{2}^{s+\frac{n-d}{2}}\left(\mathbb{R}^{n}\right): \operatorname{tr}_{\Gamma} f=0\right\} \oplus \operatorname{ext}_{\Gamma, s} B_{2}^{s}(\Gamma)
$$

in self-explaining notation. We refer for more details to [42, section 9.34(vii), pp. 157$158]$. 


\subsection{Quarkonial characterizations}

In the sections 2 and 3 in [42] we introduced the spaces $B_{p q}^{s}\left(\mathbb{R}^{n}\right)$ and $F_{p q}^{s}\left(\mathbb{R}^{n}\right)$ for all admitted parameters $s, p, q$ in terms of quarkonial representations and proved afterwards (in the same sections) that they coincide with the usual Fourier-analytical definitions of these spaces. This applies in particular to the special spaces $B_{p}^{s}\left(\mathbb{R}^{n}\right)$ according to (5) with $s>0$ and $1<p<\infty$. Quarkonial representations rely on the wavelet philosophy: functions are represented by sums over elementary building blocks which are translated and dyadically dilated according to the (pure) lattices $2^{-j} \mathbb{Z}^{n}$ in $\mathbb{R}^{n}$ with $j \in \mathbb{N}_{0}$ and where $\mathbb{Z}^{n}$ is the standard lattice in $\mathbb{R}^{n}$ consisting of all points in $\mathbb{R}^{n}$ with integer-valued components. One advantage of the quarkonial approach is the high flexibility of the underlying lattices. In particular one can replace the pure lattices $2^{-j} \mathbb{Z}^{n}$ by approximate lattices optimally adapted to a given compact set $\Gamma$ in $\mathbb{R}^{n}$. We indicated this possibility in $[42$, section 2.14, p. 25, 26], and used it in [42, sections 9.29-9.33, pp. 148-152]. We modify now this approach and adapt it to our later needs.

We always assume that $\Gamma$ is a compact $d$-set in $\mathbb{R}^{n}$ with $0<d<n$ according to Definition 2.3. Let for $\delta>0$,

$$
\Gamma_{\delta}=\left\{x \in \mathbb{R}^{n}: \operatorname{dist}(x, \Gamma)<\delta\right\}
$$

be an $\delta$-neighborhood of $\Gamma$. Let $\varepsilon>0$ be fixed (later on $\varepsilon$ will be the same number as in (2) and (3), in particular $0<\varepsilon<1$ ). Let for $k \in \mathbb{N}_{0}$,

$$
\left\{\gamma^{k, m}\right\}_{m=1}^{M_{k}} \subset \Gamma \quad \text { and } \quad\left\{\psi^{k, m}\right\}_{m=1}^{M_{k}}
$$

be (approximate) lattices and subordinated resolutions of unity with the following properties:

(i) For some $c_{1}>0$,

$$
\left|\gamma^{k, m_{1}}-\gamma^{k, m_{2}}\right| \geq c_{1} 2^{-\varepsilon k}, \quad k \in \mathbb{N}_{0}, \quad m_{1} \neq m_{2} .
$$

(ii) For some $k_{0} \in \mathbb{N}$, some $c_{2}>0$ and $\delta_{k}=c_{2} 2^{-\varepsilon k}$,

$$
\Gamma_{\delta_{k}} \subset \bigcup_{m=1}^{M_{k}} B\left(\gamma^{k, m}, 2^{-\varepsilon\left(k+2 k_{0}\right)}\right), \quad k \in \mathbb{N}_{0},
$$

where again $B(x, r)$ are $\mathbb{R}^{n}$-balls centered at $x \in \mathbb{R}^{n}$ and of radius $r>0$.

(iii) Furthermore, $\psi^{k, m}$ are non-negative $C^{\infty}$ functions in $\mathbb{R}^{n}$ with

$$
\begin{gathered}
\operatorname{supp} \psi^{k, m} \subset B\left(\gamma^{k, m}, 2^{-\varepsilon\left(k+k_{0}\right)}\right), \quad k \in \mathbb{N}_{0}, \quad m=1, \ldots, M_{k}, \\
\left|D^{\alpha} \psi^{k, m}(x)\right| \leq c_{\alpha} 2^{k \varepsilon|\alpha|}, \quad k \in \mathbb{N}_{0}, \quad m=1, \ldots, M_{k},
\end{gathered}
$$


for all multi-indices $\alpha \in \mathbb{N}_{0}^{n}$ and suitable constants $c_{\alpha}$, and

$$
\sum_{m=1}^{M_{k}} \psi^{k, m}(x)=1, \quad k \in \mathbb{N}_{0}, \quad x \in \Gamma_{\delta_{k}} .
$$

Since $\Gamma$ is a $d$-set we may assume that $M_{k} \sim 2^{\epsilon k d}$. We refer for further explanations to $[42$, pp. 144,145$]$. In particular one may assume that the resolution of unity $(17)$ can be extended consistently to a corresponding resolution of unity in $\mathbb{R}^{n}$. As usual we put

$$
\gamma^{\beta}=\gamma_{1}^{\beta_{1}} \cdots \gamma_{n}^{\beta_{n}} \quad \gamma \in \Gamma \subset \mathbb{R}^{n} \quad \text { and } \quad \beta \in \mathbb{N}_{0}^{n}
$$

and abbreviate

$$
B_{k, m}=B\left(\gamma^{k, m}, 2^{-\varepsilon k}\right), \quad k \in \mathbb{N}_{0}, \quad m=1, \ldots, M_{k} .
$$

Definition 2.11. Let $\Gamma$ be a compact $d$-set in $\mathbb{R}^{n}$ with $0<d<n$ and let $\varepsilon>0$.

(i) Let $\left\{\gamma^{k, m}\right\}_{m=1}^{M_{k}}$ and $\left\{\psi^{k, m}\right\}_{m=1}^{M_{k}}$ be as above. Then

$$
\varepsilon-\Psi_{\Gamma}=\left\{\psi_{\beta}^{k, m}: \beta \in \mathbb{N}_{0}^{n}, k \in \mathbb{N}_{0}, m=1, \ldots, M_{k}\right\}
$$

with

$$
\psi_{\beta}^{k, m}(\gamma)=\mathcal{H}_{\Gamma}^{d}\left(B_{k, m}\right)^{-|\beta| / d}\left(\gamma-\gamma^{k, m}\right)^{\beta} \psi^{k, m}(\gamma), \quad \gamma \in \Gamma,
$$

and for $s>0,1<p<\infty$,

$$
\varepsilon-\Psi_{\Gamma}^{s, p}=\left\{(\beta-q u)_{k m}^{\Gamma}: \beta \in \mathbb{N}_{0}^{n}, k \in \mathbb{N}_{0}, m=1, \ldots, M_{k}\right\}
$$

with the $(s, p)$ - $\beta$-quarks on $\Gamma$,

$$
(\beta-q u)_{k m}^{\Gamma}(\gamma)=\mathcal{H}_{\Gamma}^{d}\left(B_{k, m}\right)^{\frac{s}{d}-\frac{1}{p}} \psi_{\beta}^{k, m}(\gamma), \quad \gamma \in \Gamma .
$$

(ii) Let

$$
\nu=\left\{\nu_{k m}^{\beta} \in \mathbb{C}: \beta \in \mathbb{N}_{0}^{n}, k \in \mathbb{N}_{0}, m=1, \ldots, M_{k}\right\}
$$

and $1<p<\infty$. Then

$$
\ell_{p}^{\Gamma}=\left\{\nu:\left\|\nu \mid \ell_{p}^{\Gamma}\right\|=\left(\sum_{\beta, k, m}\left|\nu_{k m}^{\beta}\right|^{p}\right)^{1 / p}<\infty\right\} .
$$

Remark 2.12. Of course, $\mathbb{C}$ is the complex plane. By the above normalization we have for suitably chosen $k_{0}$ in (14), (15) (in dependence on the equivalence constants in (6)) for some $c>0$ the exponential decay

$$
\left|\psi_{\beta}^{k, m}(\gamma)\right| \leq c 2^{-\varepsilon|\beta|}, \quad \beta \in \mathbb{N}_{0}^{n}, \quad k \in \mathbb{N}_{0}, \quad m=1, \ldots, M_{k} .
$$


The $(s, p)$ - $\beta$-quarks $(\beta \text {-qu })_{k m}^{\Gamma}$ are the elementary building blocks we are looking for. It is an adapted and modified version of [42, Definition 9.27, p. 147]. There one finds also further discussions. Of course, $(\beta-\mathrm{qu})_{\mathrm{km}}^{\Gamma}$ depends on $s$ and $p$, but this will not be indicated. By (6) one can replace $\psi_{\beta}^{k, m}$ and $(\beta \text {-qu })_{k m}^{\Gamma}$ by

$$
\psi_{\beta}^{k, m} \Longleftrightarrow 2^{\varepsilon k|\beta|}\left(\gamma-\gamma^{k, m}\right)^{\beta} \psi^{k, m}(\gamma)
$$

and

$$
(\beta-\mathrm{qu})_{k m}^{\Gamma} \Longleftrightarrow 2^{-\varepsilon k(s-d / p)+\varepsilon k|\beta|}\left(\gamma-\gamma^{k, m}\right)^{\beta} \psi^{k, m}(\gamma) .
$$

But the above version might be more transparent when switching from $d$-sets to (abstract) $d$-spaces. If one uses the right-hand sides of (24) and (25), then one may choose $k_{0}=1$ in (14) and (15) what coincides with [42].

Remark 2.13. Compared with the well-known wavelet expansions (or also with atomic decompositions) the above building blocks have some advantages: they adapt easily and naturally to rough structures such as $d$-sets $\Gamma$ and they are very simple. The price to pay is the additional summation over $\beta$. But this is harmless and can always be neglected in estimates since we have the exponential decay (23).

Theorem 2.14. Let $\Gamma$ be a compact $d$-set in $\mathbb{R}^{n}$ with $0<d<n$ according to Definition 2.3. Let $B_{p}^{s}(\Gamma)$ be the spaces as introduced in Definition 2.7 where $s>0$ and $1<p<\infty$. Let $\varepsilon>0$ and let $(\beta \text {-qu })_{k m}^{\Gamma}$ be the corresponding $(s, p)$ - $\beta$-quarks on $\Gamma$ in (20). Let $\ell_{p}^{\Gamma}$ be the sequence spaces according to Definition 2.11 (ii).

(i) Then $B_{p}^{s}(\Gamma)$ is the collection of all $f \in L_{1}(\Gamma)$ which can be represented as

$$
f(\gamma)=\sum_{\beta \in \mathbb{N}_{0}^{n}} \sum_{k=0}^{\infty} \sum_{m=1}^{M_{k}} \nu_{k m}^{\beta}(\beta-q u)_{k m}^{\Gamma}(\gamma), \quad\left\|\nu \mid \ell_{p}^{\Gamma}\right\|<\infty,
$$

$\gamma \in \Gamma$ (absolute convergence being in $L_{1}(\Gamma)$ ). Furthermore,

$$
\left\|f\left|B_{p}^{s}(\Gamma)\|\sim \inf \| \nu\right| \ell_{p}^{\Gamma}\right\|,
$$

where the infimum is taken over all admissible representations (26).

(ii) In addition, let $\mathbb{N}_{0} \ni K<s<K+1$. There is a linear and bounded mapping

$$
f \mapsto \nu(f)=\left\{\nu_{k m}^{\beta}(f)\right\}: \quad B_{p}^{s}(\Gamma) \mapsto \ell_{p}^{\Gamma}
$$

such that

$$
f(\gamma)=\sum_{\beta, k, m} \nu_{k m}^{\beta}(f)(\beta-\mathrm{qu})_{k m}^{\Gamma}(\gamma)
$$

with

$$
\left\|f\left|B_{p}^{s}(\Gamma)\|\sim\| \nu(f)\right| \ell_{p}^{\Gamma}\right\|
$$

(equivalent norms where the equivalence constants do not depend on $f$ ). 
Remark 2.15. Part (i) with $\varepsilon=1$ and the modified $(s, p)$ - $\beta$-quarks as indicated in $(24)$, (25) is covered by [42, Theorem 9.33 , p. 151]. In particular, the series in (26) converges absolutely in $L_{1}(\Gamma)$ if $\left\|\nu \mid \ell_{p}^{\Gamma}\right\|<\infty$ (as supposed). Hence (26) and (27) converge unconditionally in the spaces considered. The step from $\varepsilon=1$ to $\varepsilon>0$ is a technical matter and one has only to adapt the formulations and normalizing factors. If $\mathbb{N}_{0} \ni$ $K<s<K+1$ then one can apply the common extension operator $\operatorname{ext}_{\Gamma}^{K} f$ according to Proposition 2.9. One has for $g=\operatorname{ext}_{\Gamma}^{K} f$ in $B_{p}^{\sigma}\left(\mathbb{R}^{n}\right)$ with $\sigma=s+\frac{n-d}{p}$ optimal quarkonial decompositions

$$
g=\sum_{\beta \in \mathbb{N}_{0}^{n}} \sum_{k=0}^{\infty} \sum_{m \in \mathbb{Z}^{n}} \lambda_{k m}^{\beta}(g)(\beta-\mathrm{qu})_{k m}
$$

where $(\beta \text {-qu })_{k m}$ are $(\sigma, p)$ - $\beta$-quarks in $\mathbb{R}^{n}, \lambda_{k m}^{\beta}(g)$ depend linearly on $g$ and

$$
\left\|g \mid B_{p}^{\sigma}\left(\mathbb{R}^{n}\right)\right\| \sim\left(\sum_{\beta, k, m}\left|\lambda_{k m}^{\beta}(g)\right|^{p}\right)^{1 / p} .
$$

This applies not only to pure lattices $2^{-k} \mathbb{Z}^{n}$ but also to approximate lattices in $\mathbb{R}^{n}$ containing the above approximate lattices on $\Gamma$. We refer to $[42,2.14-2.16$, pp. 25-27]. Then one gets part (ii) of the above theorem. This may justify to refer to $(27)$ as a frame representation and to call $\varepsilon-\Psi_{\Gamma}^{s, p}$ a frame consisting of normalized $(s, p)$ - $\beta$-quarks.

\subsection{Atomic characterizations}

Atomic representations in function spaces have some history which may be found in $\left[40\right.$, section 1.9]. Atoms in $B_{p q}^{s}\left(\mathbb{R}^{n}\right)$ and $F_{p q}^{s}\left(\mathbb{R}^{n}\right)$ go back essentially to [13-15] (but one may consult [40, section 1.9], for a more balanced history). As for more details, proofs, and recent formulations we refer to [41, section 13]. It is the main aim of this subsection to present a new atomic representation theorem for the spaces $B_{p}^{s}(\Gamma)$ according to Definition 2.7 based on intrinsic atoms. But first we describe the outcome when one simply reduces smooth atomic representation in $B_{p}^{\sigma}\left(\mathbb{R}^{n}\right)$ with $\sigma=s+\frac{n-d}{p}$ to $B_{p}^{s}(\Gamma)$, adapted to our later needs.

Let $B_{k, m}$ be the same balls as in (18) based on the $2^{-\varepsilon k}$-lattices for the $d$-set $\Gamma$ according to (13)-(14). Let

$$
s>0, \quad 1<p<\infty, \quad \text { and } \quad \sigma=s+\frac{n-d}{p}<N \in \mathbb{N} .
$$

Then $a_{\Gamma}^{k, m}$, defined on $\mathbb{R}^{n}$, is called a smooth $(s, p)$-atom (more precisely a smooth $(s, p)$ - $\varepsilon$-atom) on $\Gamma$ if

$$
\operatorname{supp} a_{\Gamma}^{k, m} \subset B_{k, m}, \quad k \in \mathbb{N}_{0}, \quad m=1, \ldots, M_{k},
$$


and

$$
\left|D^{\alpha} a_{\Gamma}^{k, m}(x)\right| \leq \mathcal{H}_{\Gamma}^{d}\left(B_{k, m}\right)^{\frac{s}{d}-\frac{1}{p}-\frac{|\alpha|}{d}}, \quad x \in \mathbb{R}^{n}, \quad|\alpha| \leq N,
$$

where the latter conditions can be re-written as

$$
\left|D^{\alpha} a_{\Gamma}^{k, m}(x)\right| \leq c 2^{-\varepsilon k\left(s-\frac{d}{p}-|\alpha|\right)}=c 2^{-\varepsilon k\left(\sigma-\frac{n}{p}-|\alpha|\right)} .
$$

Hence, $a_{\Gamma}^{k, m}$ are also smooth $(\sigma, p)$-atoms in $\mathbb{R}^{n}$ without moment conditions with respect to approximate lattices of mesh length $2^{-\varepsilon k}$. (In [41, section 13], and also in the quoted literature one relies on dyadic mesh lengths $2^{-k}$. But the replacement of $2^{-k}$ by $2^{-\varepsilon k}$ is only a technical matter resulting in better adapted formulations of type $(29))$.

Proposition 2.16. Let $\Gamma$ be a compact $d$-set in $\mathbb{R}^{n}$ with $0<d<n$ according to Definition 2.3. Let $B_{p}^{s}(\Gamma)$ be the spaces as introduced in Definition 2.7. Let $\varepsilon>0$. Then $B_{p}^{s}(\Gamma)$ is the collection of all $f \in L_{1}(\Gamma)$ which can be represented as

$$
f(\gamma)=\sum_{k=0}^{\infty} \sum_{m=1}^{M_{k}} \lambda_{k m} a_{\Gamma}^{k, m}(\gamma), \quad \gamma \in \Gamma
$$

where $a_{\Gamma}^{k, m}$ are smooth $(s, p)$ - $\varepsilon$-atoms on $\Gamma$ according to (28), (29),

$$
\lambda=\left\{\lambda_{k m} \in \mathbb{C}: k \in \mathbb{N}_{0}, m=1, \ldots, M_{k}\right\}
$$

and

$$
\left\|\lambda \mid \ell_{p}^{\Gamma, 0}\right\|=\left(\sum_{k=0}^{\infty} \sum_{m=1}^{M_{k}}\left|\lambda_{k m}\right|^{p}\right)^{1 / p}<\infty
$$

(absolute convergence being in $L_{1}(\Gamma)$ ). Furthermore,

$$
\left\|f\left|B_{p}^{s}(\Gamma)\|\sim \inf \| \lambda\right| \ell_{p}^{\Gamma, 0}\right\|,
$$

where the infimum is taken over all admissible representations.

Remark 2.17. Again it follows by (28), (29), and (33) that (31) converges absolutely in $L_{1}(\Gamma)$ and, hence, unconditionally in $B_{p}^{s}(\Gamma)$. Otherwise the above proposition follows from the atomic representation theorem for $B_{p}^{\sigma}\left(\mathbb{R}^{n}\right)$ according to $[41$, section 13 , Definition 2.7], the above considerations and the comments in the following remark.

Remark 2.18. We add a technical remark which might be of interest for its own sake. In case of quarkonial representation we have (17). Hence if this resolution of unity is extended to $\mathbb{R}^{n}$ with related non-negative $C^{\infty}$ functions then these complementing functions are zero on $\Gamma$. When shifting quarkonial decompositions for $B_{p}^{\sigma}\left(\mathbb{R}^{n}\right)$ with $\sigma=s+\frac{n-d}{p}$ to $B_{p}^{s}(\Gamma)$ then only $\beta$-quarks according to Definition 2.11 contribute to this reduction and Theorem 2.14 follows from corresponding quarkonial decompositions 
for $B_{p}^{\sigma}\left(\mathbb{R}^{n}\right)$. This is not so clear in case of atoms. However one can derive optimal atomic decompositions from optimal quarkonial decompositions as follows. Let $f$ be given by (26). Then

$$
\begin{aligned}
f(\gamma) & =\sum_{k=0}^{\infty} \sum_{m=1}^{M_{k}}\left(\sum_{\eta \in \mathbb{N}_{0}^{n}} 2^{-\varepsilon^{\prime}|\eta|}\left|\nu_{k m}^{\eta}\right|\right)\left(\sum_{\beta \in \mathbb{N}_{0}^{n}} \frac{\nu_{k m}^{\beta}(\beta-q u)_{k m}^{\Gamma}(\gamma)}{\sum_{\eta \in \mathbb{N}_{0}^{n}} 2^{-\varepsilon^{\prime}|\eta|}\left|\nu_{k m}^{\eta}\right|}\right) \\
& =\sum_{k=0}^{\infty} \sum_{m=1}^{M_{k}} \lambda_{k m} a_{\Gamma}^{k, m}(\gamma),
\end{aligned}
$$

where $0<\varepsilon^{\prime}<\varepsilon$. By $(23)$ and corresponding estimates for $D^{\alpha} \psi_{\beta}^{k, m}$ with $\varepsilon^{\prime}$ in place of $\varepsilon$ one gets (28), (29) as a consequence of (15), (20). Hence, $a_{\Gamma}^{k, m}$ are smooth atoms of the above type. Furthermore by (32), (33) and (21), (22) one gets

$$
\left\|\lambda\left|\ell_{p}^{\Gamma, 0}\|\leq c\| \nu\right| \ell_{p}^{\Gamma}\right\|
$$

Hence if (26) is an optimal quarkonial decomposition then (34) is an optimal atomic decomposition. This justifies in particular Proposition 2.16.

Remark 2.19. We compare the $(s, p)$ - $\beta$-quarks $(\beta \text {-qu })_{k m}^{\Gamma}$ on a compact $d$-set $\Gamma$ in $\mathbb{R}^{n}$ according to Definition 2.11 with the corresponding smooth $(s, p)$-atoms as described in $(28)-(30)$. Although the functions $(\beta \text {-qu })_{k m}^{\Gamma}$ originate from the surrounding $\mathbb{R}^{n}$ one can accept them as intrinsic building blocks (at least) for the spaces $B_{p}^{s}(\Gamma)$ as described in Theorem 2.14. The situation might be similar as in case of wavelet bases and wavelet characterizations of function spaces. The main advantage of the Meyer wavelet bases and, even more, of the Daubechies wavelet bases in $L_{2}\left(\mathbb{R}^{n}\right)$ is their existence. We refer to [35] and [48] for details of the somewhat complicated constructions of these wavelets and for their properties. But these wavelets may also serve as building blocks for all spaces $B_{p q}^{s}\left(\mathbb{R}^{n}\right)$ and $F_{p q}^{s}\left(\mathbb{R}^{n}\right)$ for all admitted parameters $s, p, q$. We refer to [44], and also to [45] as far as the anisotropic extensions are concerned. On the other hand, one can hardly accept the restriction of (29) to $\Gamma$ as an intrinsic description of smooth $(s, p)$-atoms on $\Gamma$. In general it is not clear how a better adapted substitute of (29) might look like. But there is a satisfactory solution of this question if $0<s<1$ which we are going to discuss now and which will play a decisive role in the theory of function spaces on $d$-sets and on $d$-spaces as developed in this paper.

Definition 2.20. Let $\Gamma$ be a compact $d$-set in $\mathbb{R}^{n}$ with $0<d<n$ according to Definition 2.3. Let $\varepsilon>0,1<p<\infty$ and $0<s<1$. Let

$$
B_{k, m}^{\Gamma}=\left\{\gamma \in \Gamma:\left|\gamma-\gamma^{k, m}\right| \leq 2^{-\varepsilon k}\right\}, \quad k \in \mathbb{N}_{0}, \quad m=1, \ldots, M_{k},
$$

be the intersection of $B_{k, m}$ in (18) with $\Gamma$, where the lattices $\left\{\gamma^{k, m}\right\}$ have the same meaning as in (13)-(14). Then a Lipschitz-continuous function $a_{\Gamma}^{k, m}$ on $\Gamma$ is called an 
$(s, p)^{*}$-atom (more precisely, an $(s, p)^{*}$ - $\varepsilon$-atom) if for $k \in \mathbb{N}_{0}$ and $m=1, \ldots, M_{k}$,

$$
\begin{gathered}
\operatorname{supp} a_{\Gamma}^{k, m} \subset B_{k, m}^{\Gamma}, \\
\left|a_{\Gamma}^{k, m}(\gamma)\right| \leq \mathcal{H}_{\Gamma}^{d}\left(B_{k, m}^{\Gamma}\right)^{\frac{s}{d}-\frac{1}{p}}, \quad \gamma \in \Gamma,
\end{gathered}
$$

and

$$
\left|a_{\Gamma}^{k, m}(\gamma)-a_{\Gamma}^{k, m}(\delta)\right| \leq \mathcal{H}_{\Gamma}^{d}\left(B_{k, m}^{\Gamma}\right)^{\frac{s-1}{d}-\frac{1}{p}}|\gamma-\delta|
$$

with $\gamma \in \Gamma, \delta \in \Gamma$.

Remark 2.21. Here $|\cdot|$ has the usual double meaning as the absolute value of complex numbers and the Euclidean distance $\varrho_{n}(\gamma, \delta)=|\gamma-\delta|$ in $\mathbb{R}^{n}$. These $(s, p)^{*}$-atoms are the intrinsic counterparts of the smooth $(s, p)$-atoms according to (28) and (29) with $|\alpha|=1$. In particular, the restriction of any smooth $(s, p)$-atom to $\Gamma$ is an $(s, p)^{*}$-atom on $\Gamma$. It is one of the main aims of this section to prove the counterpart of Proposition 2.16 for the spaces $B_{p}^{s}(\Gamma)$ where $1<p<\infty$ and $0<s<1$ with $(s, p)^{*}$-atoms in place of $(s, p)$-atoms. For this purpose we need some preparations. First we recall that $B_{p}^{s}(\Gamma)$ can be equivalently normed by

$$
\left\|\left.f\left|B_{p}^{s}(\Gamma) \|_{*}^{p}=\int_{\Gamma}\right| f(\gamma)\right|^{p} \mu(\mathrm{d} \gamma)+\int_{\Gamma \times \Gamma} \frac{|f(\gamma)-f(\delta)|^{p}}{|\gamma-\delta|^{d+s p}} \mu(\mathrm{d} \gamma) \mu(\mathrm{d} \delta)\right.
$$

with $\mu=\mathcal{H}_{\Gamma}^{d}$ for short. We refer to [27, chapter V]. In particular, $a_{\Gamma}^{k, m} \in B_{p}^{s}(\Gamma)$ for the above $(s, p)^{*}$-atoms for all $1<p<\infty$ and $0<s<1$. Let $0<c_{1}<c_{2}$ and $0<c_{3}<c_{4}$ be four given numbers and let $\Gamma$ be $d$-sets in $\mathbb{R}^{n}$ with

$$
\operatorname{supp} \Gamma \subset\{x:|x|<1\}, \quad c_{1} \leq \mu(\Gamma) \leq c_{2},
$$

and

$$
c_{3} r^{d} \leq \mu(B(\gamma, r)) \leq c_{4} r^{d} \quad \text { for } \quad \gamma \in \Gamma, \quad 0<r \leq \operatorname{diam} \Gamma,
$$

as in (6). Then the norm of the extension operator $\operatorname{ext}_{\Gamma}=\operatorname{ext}_{\Gamma}^{0}$ from Proposition 2.9,

$$
\operatorname{ext}_{\Gamma}: \quad B_{p}^{s}(\Gamma) \hookrightarrow B_{p}^{\sigma}\left(\mathbb{R}^{n}\right), \quad 0<s<1,1<p<\infty, \sigma=s+\frac{n-d}{p},
$$

can be estimated from above uniformly for all these $d$-sets $\Gamma$, all $s$ and all $p$. This follows from the proof of Theorem 1 in [27, p. 103].

Proposition 2.22. Let $\Gamma$ be a compact d-set in $\mathbb{R}^{n}$ with $0<d<n$. Let for $0<r<1$,

$$
B^{\Gamma}(r)=\left\{\gamma \in \Gamma:\left|\gamma-\gamma^{0}\right|<r\right\} \quad \text { for some } \gamma^{0} \in \Gamma,
$$

and

$$
B(2 r)=\left\{x \in \mathbb{R}^{n}:\left|x-\gamma^{0}\right|<2 r\right\}
$$


Let $\mu=\mathcal{H}_{\Gamma}^{d}$ for short,

$$
1<p<\infty, \quad 0<s<1, \quad \sigma=s+\frac{n-d}{p} .
$$

Then

$$
\begin{aligned}
\left\|\left.f\left|B_{p}^{s}(\Gamma) \|^{p} \sim r^{-s p} \int_{B^{\Gamma}(r)}\right| f(\gamma)\right|^{p} \mu(\mathrm{d} \gamma)\right. \\
+\int_{\gamma, \delta \in B^{\Gamma}(2 r)} \frac{|f(\gamma)-f(\delta)|^{p}}{|\gamma-\delta|^{d+s p}} \mu(\mathrm{d} \gamma) \mu(\mathrm{d} \delta)
\end{aligned}
$$

for all

$$
f \in B_{p}^{s}(\Gamma) \quad \text { with } \quad \operatorname{supp} f \subset B^{\Gamma}(r)
$$

where the equivalence constants in (44) are independent of $B^{\Gamma}(r)$ and $f$. Furthermore,

$$
\left\|f\left|B_{p}^{s}(\Gamma)\|\sim \inf \| g\right| B_{p}^{\sigma}\left(\mathbb{R}^{n}\right)\right\|,
$$

where the infimum is taken over all

$$
g \in B_{p}^{\sigma}\left(\mathbb{R}^{n}\right), \quad g \mid \Gamma=f, \quad \operatorname{supp} g \subset B(2 r) .
$$

The equivalence constants in (46) are independent of $B^{\Gamma}(r)$ and $f$ with (45).

Proof. Step 1. First we prove (44) using (38). We may assume $r=2^{-k}$ for some $k \in \mathbb{N}$. Then

$$
\begin{aligned}
\int_{\Gamma \times \Gamma} \frac{|f(\gamma)-f(\delta)|^{p}}{|\gamma-\delta|^{d+s p}} \mu(\mathrm{d} \gamma) \mu(\mathrm{d} \delta) & \sim \int_{\gamma, \delta \in B^{\Gamma}(2 r)} \frac{|f(\gamma)-f(\delta)|^{p}}{|\gamma-\delta|^{d+s p}} \mu(\mathrm{d} \gamma) \mu(\mathrm{d} \delta) \\
& +\int_{\gamma \in B^{\Gamma}(r)}|f(\gamma)|^{p} \int_{|\delta-\gamma| \geq 2^{-k}} \frac{\mu(\mathrm{d} \delta)}{|\gamma-\delta|^{d+s p}} \mu(\mathrm{d} \gamma) .
\end{aligned}
$$

The inner integral over $|\delta-\gamma| \geq 2^{-k}$ is equivalent to

$$
\sum_{l=0}^{L} 2^{(k-l)(d+s p)} 2^{d(l-k)} \sim 2^{k s p} \sim r^{-s p} .
$$

Then (44) with (45) is a consequence of (38), (48).

Step 2. We prove (46) with (47) for $f$ according to (45). We may assume $\gamma^{0}=0$ in (42), (43) and $r=2^{-k}$ for some $k \in \mathbb{N}$. Obviously, $B^{\Gamma}\left(2^{-k}\right)$ is again a $d$-set with total mass $\mu\left(B^{\Gamma}\left(2^{-k}\right)\right) \sim 2^{-k d}$. Let

$$
D_{k}: x \mapsto 2^{k} x, \quad x \in \mathbb{R}^{n},
$$


be a dyadic dilation mapping in particular the $d$-set $B^{\Gamma}\left(2^{-k}\right)$ onto the $d$-set

$$
\Gamma_{k}=D_{k} B^{\Gamma}\left(2^{-k}\right) \quad \text { with the measure } \mu_{k}=2^{k d} \mu \circ D_{k}^{-1} .
$$

Here $\mu^{k}=\mu \circ D_{k}^{-1}$ is the image measure and the factor $2^{k d}$ compensates the total mass $\mu^{k}\left(\Gamma_{k}\right) \sim 2^{-k d}$. Then we may assume that all $d$-sets $\Gamma_{k}$ fit in the scheme (39), (40). Let

$$
\tilde{f}(\tilde{\gamma})=f(\gamma) \quad \gamma=2^{-k} \tilde{\gamma}, \quad \tilde{\gamma} \in \Gamma_{k},
$$

be the transferred functions with (45). Then it follows by (44) with $r=2^{-k}$,

$$
\begin{aligned}
& \left\|f \mid B_{p}^{s}(\Gamma)\right\|^{p} \\
& \sim 2^{k s p} \int_{\Gamma_{k}}|\tilde{f}(\tilde{\gamma})|^{p} \mu^{k}(\mathrm{~d} \tilde{\gamma})+2^{k(d+s p)} \int_{\Gamma_{k} \times \Gamma_{k}} \frac{|\tilde{f}(\tilde{\gamma})-\tilde{f}(\tilde{\delta})|^{p}}{|\tilde{\gamma}-\tilde{\delta}|^{d+s p}} \mu^{k}(\mathrm{~d} \tilde{\gamma}) \mu^{k}(\mathrm{~d} \tilde{\delta}) \\
& \sim 2^{k(s p-d)}\left[\int_{\Gamma_{k}}|\tilde{f}(\tilde{\gamma})|^{p} \mu_{k}(\mathrm{~d} \tilde{\gamma})+\int_{\Gamma_{k} \times \Gamma_{k}} \frac{|\tilde{f}(\tilde{\gamma})-\tilde{f}(\tilde{\delta})|^{p}}{|\tilde{\gamma}-\tilde{\delta}|^{d+s p}} \mu_{k}(\mathrm{~d} \tilde{\gamma}) \mu_{k}(\mathrm{~d} \tilde{\delta})\right] \\
& \sim 2^{k(s p-d)}\left\|\tilde{f} \mid B_{p}^{s}\left(\Gamma_{k}\right)\right\|^{p}
\end{aligned}
$$

where all equivalence constants are independent of $k$ and $f$ with (45). Since (39)-(41) can be applied it follows

$$
\left\|\tilde{f}\left|B_{p}^{s}\left(\Gamma_{k}\right)\|\sim \inf \| \tilde{g}\right| B_{p}^{\sigma}\left(\mathbb{R}^{n}\right)\right\|,
$$

where the infimum is taken over all

$$
\tilde{g} \in B_{p}^{\sigma}\left(\mathbb{R}^{n}\right) \quad \text { with } \quad \tilde{g} \mid \Gamma_{k}=\tilde{f} \quad \text { and } \quad \operatorname{supp} \tilde{g} \subset\{x:|x|<2\} .
$$

Using the counterpart of (49), hence

$$
\tilde{g}(\tilde{x})=g(x) \quad \text { with } \quad x=2^{-k} \tilde{x}, \quad|\tilde{x}|<2,
$$

then one obtains from [42, Corollary 5.16, p. 66] that

$$
\left\|g\left|B_{p}^{\sigma}\left(\mathbb{R}^{n}\right)\left\|\sim 2^{k(\sigma-n / p)}\right\| \tilde{g}\right| B_{p}^{\sigma}\left(\mathbb{R}^{n}\right)\right\|=2^{k(s-d / p)}\left\|\tilde{g} \mid B_{p}^{\sigma}\left(\mathbb{R}^{n}\right)\right\| .
$$

But now (46), (47) follows from (51), the homogeneity relations (50), (54) and (52), (53).

Theorem 2.23. Let $\Gamma$ be a compact $d$-set in $\mathbb{R}^{n}$ with $0<d<n$ according to Definition 2.3. Let $B_{p}^{s}(\Gamma)$ with $1<p<\infty, 0<s<1$ be the spaces as introduced in Definition 2.7. Let $\varepsilon>0$. Then $B_{p}^{s}(\Gamma)$ is the collection of all $g \in L_{1}(\Gamma)$ which can be represented as

$$
f(\gamma)=\sum_{k=0}^{\infty} \sum_{m=1}^{M_{k}} \lambda_{k m} a_{\Gamma}^{k, m}(\gamma), \quad \gamma \in \Gamma, \quad\left\|\lambda \mid \ell_{p}^{\Gamma, 0}\right\|<\infty
$$


where $a_{\Gamma}^{k, m}$ are the $(s, p)^{*}-\varepsilon$-atoms on $\Gamma$ according to Definition 2.20 and where $\lambda$ and $\left\|\lambda \mid \ell_{p}^{\Gamma, 0}\right\|$ have the same meaning as in (32), (33). Furthermore,

$$
\left\|f\left|B_{p}^{s}(\Gamma)\|\sim \inf \| \lambda\right| \ell_{p}^{\Gamma, 0}\right\|
$$

where the infimum is taken over all admissible representations (55).

Proof. By Proposition 2.16 we have (55) and (56) for the smooth $(s, p)$ - $\varepsilon$-atoms which are special $(s, p)^{*}-\varepsilon$-atoms as mentioned at the beginning of Remark 2.21. Hence one has to show that this representation can be extended to all $(s, p)^{*}$ - $\varepsilon$-atoms. By (36), (37) compared with (29), $|\alpha|=0$ and $|\alpha|=1$, it follows that the $(s, p)^{*}$ - $\varepsilon$-atoms are correctly normalized. But it seems to be reasonable to make this point more explicit by direct calculations. This will be done in Remark 2.24 below. Hence there is a constant $c$ such that for all these atoms

$$
\left\|a_{\Gamma}^{k, m} \mid B_{p}^{s}(\Gamma)\right\| \leq c .
$$

Furthermore for different values of $s$ the corresponding $(s, p)^{*}$ - $\varepsilon$-atoms differ only by their normalizing factors. As a consequence one gets for the above $(s, p)^{*}-\varepsilon$-atoms,

$$
\left\|a_{\Gamma}^{k, m} \mid B_{p}^{\tilde{s}}(\Gamma)\right\| \leq \mathcal{H}_{\Gamma}^{d}\left(B_{k m}^{\Gamma}\right)^{\frac{s-\tilde{s}}{d}} \sim r^{s-\tilde{s}},
$$

where $r=2^{-\varepsilon k}$ and $0<s \leq \tilde{s}<1$. By Proposition 2.22 and its proof it follows that any $(s, p)^{*}-\varepsilon$-atom can be extended to a function

$$
a^{k m} \in B_{p}^{\tilde{\sigma}}\left(\mathbb{R}^{n}\right) \quad \text { where } \quad \tilde{\sigma}=\tilde{s}+\frac{n-d}{p}, \quad 0<s \leq \tilde{s}<1
$$

such that for $r=2^{-\varepsilon k}$,

$$
a^{k m} \mid \Gamma=a_{\Gamma}^{k m}, \quad \operatorname{supp} a^{k m} \subset\left\{x \in \mathbb{R}^{n}:\left|x-\gamma^{k, m}\right| \leq c_{1} r\right\}
$$

and

$$
\left\|a^{k m} \mid B_{p}^{\tilde{\sigma}}\left(\mathbb{R}^{n}\right)\right\| \leq c_{2} r^{s-\tilde{s}}
$$

where $c_{1}$ and $c_{2}$ are positive constants which are independent of $r, \tilde{s}$ with $1>\tilde{s} \geq$ $s$, and the atoms $a_{\Gamma}^{k m}$. Then $a^{k m}$ are non-smooth atoms in $B_{p}^{\tilde{\sigma}}\left(\mathbb{R}^{n}\right)$ according to [43, Definition 2, p. 465]. Then one gets the above theorem as in Proposition 2.16 now based on the non-smooth atomic representation Theorem 2 in [43, p. 465].

Remark 2.24. We give an explicit proof of (57), what covers also (58) by the arguments between these two formulas. We rely on (44) with $f=a_{\Gamma}^{k m}$ and $r \sim 2^{-\varepsilon k}$. Then it follows from (36),

$$
r^{-s p} \int_{B^{\Gamma}(r)}|f(\gamma)|^{p} \mu(\mathrm{d} \gamma) \leq c r^{-s p} r^{s p-d} r^{d}=c
$$


and from (37) (assuming that $B^{\Gamma}(r)$ is centered at the origin)

$$
\begin{gathered}
\int_{\gamma, \delta \in B^{\Gamma}(r)} \frac{|f(\gamma)-f(\delta)|^{p}}{|\gamma-\delta|^{d+s p}} \mu(\mathrm{d} \gamma) \mu(\mathrm{d} \delta) \\
\leq c r^{(s-1) p-d} \int_{\gamma, \delta \in B^{\Gamma}(r)}|\gamma-\delta|^{(1-s) p-d} \mu(\mathrm{d} \gamma) \mu(\mathrm{d} \delta) \\
\leq c r^{(s-1) p-d} \int_{|\gamma| \leq c^{\prime} r|\delta| \leq c^{\prime} r}|\delta|^{(1-s) p-d} \mu(\mathrm{d} \delta) \mu(\mathrm{d} \gamma) \\
\leq c^{\prime \prime} r^{(s-1) p-d} r^{d} r^{(1-s) p}=c^{\prime \prime} .
\end{gathered}
$$

This proves (57).

\section{Quasi-metric spaces}

We summarized the main aim of this paper in the introductory section 1 , especially in the formulas (2), (3): the transference of spaces of type $B_{p}^{s}$ from some (concrete) $d^{\prime}$-sets in some $\mathbb{R}^{n}$ via snowflaked transforms to corresponding spaces on (abstract) $d$ spaces, which are special (abstract) complete quasi-metric spaces. So far we developed in section 2 the theory of the spaces $B_{p}^{s}(\Gamma)$ on $d$-sets $\Gamma$ in $\mathbb{R}^{n}$ adapted to our purpose. In this section we describe the basic ingredients about quasi-metric spaces and $d$ spaces needed later on.

\subsection{Basic assertions}

Let $X$ be a set. A non-negative function $\varrho(x, y)$ defined on $X \times X$ is called a quasimetric if it has the following properties:

$$
\begin{aligned}
\varrho(x, y)=0 & \text { if, and only if, } \quad x=y, \\
\varrho(x, y)=\varrho(y, x) & \text { for all } x \in X \text { and all } y \in X,
\end{aligned}
$$

there is a real number $A$ with $A \geq 1$ such that for all $x, y, z \in X$,

$$
\varrho(x, y) \leq A[\varrho(x, z)+\varrho(z, y)]
$$

If $A=1$ is admissible, then $\varrho$ is a metric and (59) is the triangle inequality. As usual, a quasi-metric $\varrho$ on $X$ is said to be equivalent to $\varrho$, written as $\varrho \sim \bar{\varrho}$, if there are two positive numbers $c_{1}$ and $c_{2}$ such that

$$
c_{1} \varrho(x, y) \leq \bar{\varrho}(x, y) \leq c_{2} \varrho(x, y), \quad x \in X, \quad y \in X
$$


Theorem 3.1. Let $\varrho$ be a quasi-metric on a set $X$.

(i) There is a number $\varepsilon_{0}$ with $0<\varepsilon_{0} \leq 1$ such that $\varrho^{\varepsilon}$ for any $\varepsilon$ with $0<\varepsilon \leq \varepsilon_{0}$ is equivalent to a metric.

(ii) Let $0<\varepsilon \leq \varepsilon_{0}$ and let $\varrho \sim \varrho$ such that $\varrho^{\varepsilon}$ is a metric according to part (i). Then there is a positive number $c$ such that for all $x \in X, y \in X, z \in X$,

$$
|\bar{\varrho}(x, y)-\bar{\varrho}(x, z)| \leq c \bar{\varrho}(y, z)^{\varepsilon}[\bar{\varrho}(x, y)+\bar{\varrho}(x, z)]^{1-\varepsilon} .
$$

Remark 3.2. Part (i) is a remarkable observation. A proof may be found in [23, Proposition 14.5 , p. 110-112]. The assertion is even slightly stronger: If $\varrho \sim \bar{\varrho}$ such that $\bar{\varrho}^{\varepsilon_{0}}$ is a metric, then $\varrho^{\varepsilon}$ is also a metric for any $0<\varepsilon \leq \varepsilon_{0}$. This follows from the triangle inequality for metrics and the well-known observation

$$
(a+b)^{p} \leq a^{p}+b^{p} \quad \text { where } \quad a \geq 0, \quad b \geq 0, \quad 0<p \leq 1 .
$$

Part (ii) is a consequence of part (i) and the mean value theorem for the function $t \mapsto t^{1 / \varepsilon}$, where $t>0$ :

$$
\begin{aligned}
\mid \varrho^{\varepsilon}(x, y)^{1 / \varepsilon} & -\bar{\varrho}^{\varepsilon}(x, z)^{1 / \varepsilon} \mid \\
& \leq\left|\bar{\varrho}^{\varepsilon}(x, y)-\bar{\varrho}^{\varepsilon}(x, z)\right| \varepsilon^{-1}\left(\bar{\varrho}^{\varepsilon\left(\frac{1}{\varepsilon}-1\right)}(x, y)+\bar{\varrho}^{\varepsilon\left(\frac{1}{\varepsilon}-1\right)}(x, z)\right) \\
& \leq c \bar{\varrho}(y, z)^{\varepsilon}[\bar{\varrho}(x, y)+\varrho(x, z)]^{1-\varepsilon} .
\end{aligned}
$$

Independently of part (i) the inequality (60) is known since some time. We refer to [31, Theorem 2, p. 259]. It is a corner-stone of the analysis on quasi-metric spaces and paves the way to introduce a topology on $X$ taking the balls

$$
B(x, r)=\{y \in X: \bar{\varrho}(x, y)<r\}
$$

as a basis of neighborhoods of $x \in X$, where $r>0$.

Definition 3.3. Let $\varrho$ be a quasi-metric on a set $X$ equipped with the topology as just indicated.

(i) Then $(X, \varrho, \mu)$ is called a space of homogeneous type if $\mu$ is a non-negative regular Borel measure on $X$ such that there is a constant $A$ with

$$
0<\mu(B(x, 2 r)) \leq A \mu(B(x, r)) \quad \text { for all } x \in X, r>0,
$$

(doubling condition).

(ii) Let $d>0$. Then $(X, \varrho, \mu)$ is called a $d$-space if it is a complete space of homogeneous type according to part (i) with

$$
\operatorname{diam} X=\sup \{\varrho(x, y): x \in X, y \in X\}<\infty
$$

and

$$
\mu(B(x, r)) \sim r^{d} \text { for all } x \in X \text { and } 0<r \leq \operatorname{diam} X
$$


Remark 3.4. In case of part (ii) we have

$$
\mu(\{x\})=0 \quad \text { for every } x \in X, \quad \text { and } \mu(X)<\infty .
$$

Furthermore, $\mu$ is a Radon measure and $(X, \varrho, \mu)$ is compact. As far as measuretheoretical notation is concerned we refer to $[33$, p. $7-13]$. Otherwise the above notation of $d$-spaces was introduced in [46] imitating the notation of $d$-sets in $\mathbb{R}^{n}$ according to Definition 2.3. (We added now the assumption that $d$-spaces are complete. This is convenient when it comes to snowflaked transforms onto $d^{\prime}$-sets in some $\mathbb{R}^{n}$, but quite often immaterial.) In [46, sections 2.13 and 2.14], one finds some discussions about the above definitions. As for background information we refer to $[20,21,31]$.

Remark 3.5. There has always been some interest in an analysis on spaces of homogeneous type according to Definition 3.3. We refer to [7-9]. But in the last decade, especially in the last few years there are some new developments to create a substantial intrinsic analysis on fractals and on (quasi-)metric spaces, at least partly closely connected with the problem how to measure smoothness on these structures. The underlying measures have often the property (64), but sometimes only the doubling condition (62) is required. We refer to the books $[10,23,28,36]$. As far as function spaces on spaces of homogeneous type are concerned we give some more specific references later on. However the approach presented in this paper is different from what has been done so far in literature. It is mainly based on the theory of function spaces on $d$-sets in $\mathbb{R}^{n}$ as developed in section 2 on the one hand and the following remarkable observation on the other hand.

Theorem 3.6. Let $(X, \varrho, \mu)$ be a space of homogeneous type according to Definition 3.3(i). Let $0<\varepsilon_{0} \leq 1$ be the same number as in Theorem 3.1 and let $0<\varepsilon<\varepsilon_{0}$. Then there is a natural number $n$ and a bi-Lipschitzian mapping

$$
H: X \mapsto \mathbb{R}^{n}
$$

from $\left(X, \varrho^{\varepsilon}, \mu\right)$ into $\mathbb{R}^{n}$. The dimension $n$ (and also the Lipschitzian constants) can be chosen to depend only on $\varepsilon$ and on the doubling constant $A$ in (62).

Remark 3.7. If $\varrho$ is a metric then $\left(X, \varrho^{\varepsilon}, \mu\right)$ with $\varepsilon<1$ is called the snowflaked version of $(X, \varrho, \mu)$. In case of quasi-metrics one has even to assume $\varepsilon<\varepsilon_{0}$. Then (65) means that there is a bi-Lipschitzian map $H$ of the snowflaked version $\left(X, \varrho^{\varepsilon}, \mu\right)$ onto its image in $\mathbb{R}^{n}$,

$$
|H(x)-H(y)| \sim \varrho^{\varepsilon}(x, y), \quad x \in X, \quad y \in X,
$$

where the equivalence constants are independent of $x$ and $y$. Usually this assertion is formulated and proved for metric spaces $(X, \varrho)$ with a so-called doubling metric. We refer for a definition of a doubling metric to [23, p. 81]. However if there is a doubling measure then the respective metric is also doubling, [23, p. 82]. Hence one can apply the corresponding assertions in [36, Theorem 2.2, p. 13] and [23, Theorem 12.2 on p. 98$]$ to $\left(X, \varrho^{\varepsilon_{0}}, \mu\right)$ resulting in the above theorem. The original proof of this 
remarkable assertion is due to P. Assouad [2,3]. More details may be found in $[23,36]$ as specified above and also in [10, section 5.4, p. 21].

\subsection{Euclidean charts}

We now always assume that the quasi-metric $\varrho$ of a $d$-space according to Definition 3.3 (ii) has the property (60) with $0<\varepsilon \leq \varepsilon_{0}$, where $\varepsilon_{0}$ has the same meaning as there (hence we identify $\bar{\varrho}$ with $\varrho$ ). We apply Theorem 3.6 to the snowflaked version $\left(X, \varrho^{\varepsilon}, \mu\right)$ of the $d$-space $(X, \varrho, \mu)$ with $0<\varepsilon<\varepsilon_{0}$. Consequently there is a bi-Lipschitzian map $H$,

$$
H:\left(X, \varrho^{\varepsilon}, \mu\right) \text { onto }\left(\Gamma, \varrho_{n}, \nu\right)
$$

where

$$
\varrho_{n}(x, y)=|x-y|, \quad x \in \mathbb{R}^{n}, \quad y \in \mathbb{R}^{n},
$$

is the usual Euclidean distance in $\mathbb{R}^{n}$ and $\nu$ is the image measure of $\mu$, hence $\nu=\mu \circ H^{-1}$. By (63), (66) and the assumption that $(X, \varrho, \mu)$ is complete it follows that $\Gamma=H X$ is a compact set in $\mathbb{R}^{n}$. Furthermore one gets by (64) and (66) for balls $B(\gamma, r)$ in $\mathbb{R}^{n}$ with $\gamma \in \Gamma$ and $0<r \leq 1$ that

$$
\nu(B(\gamma, r)) \sim r^{d_{\varepsilon}} \quad \text { where } \quad d_{\varepsilon}=d / \varepsilon .
$$

Hence $\left(\Gamma, \varrho_{n}, \nu\right)$ is a compact $d_{\varepsilon}$-set in some $\mathbb{R}^{n}$ according to Definition 2.3. By Remark 2.4 we may identify $\nu$ with $\mathcal{H}_{\Gamma}^{d_{\varepsilon}}$, the restriction of the Hausdorff measure $\mathcal{H}^{d_{\varepsilon}}$ in $\mathbb{R}^{n}$ to $\Gamma$.

Definition 3.8. Let $(X, \varrho, \mu)$ be a $d$-space according to Definition 3.3 and let $\varepsilon_{0}$ be the same number as in Theorem 3.1. Let $0<\varepsilon<\varepsilon_{0}$. Then any bi-Lipschitzian $\operatorname{map} H$,

$$
H:\left(X, \varrho^{\varepsilon}, \mu\right) \text { onto }\left(\Gamma, \varrho_{n}, \mathcal{H}_{\Gamma}^{d_{\varepsilon}}\right), \quad d_{\varepsilon}=d / \varepsilon,
$$

is called an Euclidean chart or an $\varepsilon$-chart of $(X, \varrho, \mu)$.

Remark 3.9. Quite obviously, there are many Euclidean charts of a given $d$-space $(X, \varrho, \mu)$. On the other hand the above restriction to $d$-spaces is by no means necessary for our purpose. In $\mathbb{R}^{n}$ one can replace $r^{d}$ in (6) by more general gauge functions $h(r)$, hence

$$
\mu(B(\gamma, r)) \sim h(r), \quad \gamma \in \Gamma, \quad 0<r \leq \operatorname{diam} \Gamma .
$$

It has been clarified in a final way in [5, Theorem 7] and $[4,6]$, which functions $h$ can be admitted. There should be an abstract counterpart of respective $h$-spaces being spaces of homogeneous type according to Definition 3.3. Then one can apply Theorem 3.6 resulting in corresponding Euclidean charts. Furthermore one may abandon (63) and deal with general complete (but not necessarily compact) $d$-spaces or $h$-spaces. Following the above procedure one might arrive at 
atlases of Euclidean charts and snowflaked bi-Lipschitzian maps.

Then one is near to the usual way as a (n-dimensional $C^{\infty}$ or Riemannian) manifold is represented by an atlas consisting of local charts.

\subsection{Snowflaked maps}

To provide a better understanding of Euclidean charts according to Definition 3.8 we illustrate the snowflaked maps $(67)$ by some examples complemented by a few comments.

Example 3.10. First we recall the well-known construction of the snowflake curve or Koch curve (1906; Niels Fabian Helge von Koch, 1870-1924, Sweden), adapted to our purpose. In the plane $\mathbb{R}^{2}$ the snowflake curve $\Gamma$ can be constructed as indicated in Figure 1 as the closure of the sequences of points (or the connecting polygonal lines)

$$
P_{k, l}, \quad \text { where } k \in \mathbb{N}_{0} \text { and } l=0, \ldots, 4^{k} \text {. }
$$

Hence, $P_{0,0}=(0,0), P_{0,1}=(1,0)$,

$$
P_{1,0}=P_{0,0}, P_{1,1}=\left(\frac{1}{3}, 0\right), P_{1,2}=\left(\frac{1}{2}, \frac{1}{2 \sqrt{3}}\right), P_{1,3}=\left(\frac{2}{3}, 0\right), P_{1,4}=P_{0,1},
$$

and so on. On the other hand we subdivide the closed unit interval $X=[0,1]$ successively in $4^{k}$ intervals with the endpoints $x_{k, l}=l \cdot 4^{-k}$, where $l=0, \ldots, 4^{k}$. Then we map $X$ continuously onto $\Gamma$,

$$
H: X \mapsto \Gamma \quad \text { with } H\left(x_{k, l}\right)=P_{k, l}
$$

where $k \in \mathbb{N}_{0}$ and $l=0, \ldots, 4^{k}$. This can be done iteratively without contradiction since

$$
P_{k+1,4 l}=P_{k, l} \quad \text { where } \quad l=0, \ldots, 4^{k},
$$

in agreement with (68). Let

$$
d=\frac{\log 4}{\log 3} \quad \text { and } \quad \varepsilon=\frac{1}{d}=\frac{\log 3}{\log 4} .
$$

First we recall that $d$ is the Hausdorff dimension of $\Gamma$ (since one needs $4^{k}$ circles of radius $3^{-k}$ to cover $\Gamma$ and $\left.4^{k} \cdot 3^{-k d}=1\right)$. We convert $X=[0,1]$ into the metric space

$$
\left(X, \varrho, \mu_{L}\right) \quad \text { with } \quad \varrho(x, y)=|x-y|^{\varepsilon}
$$

where $\mu_{L}$ is the Lebesgue measure on $\mathbb{R}$. Then one has

$$
\mu_{L}(B(x, r)) \sim r^{d}, \quad x \in X, \quad 0<r<1,
$$



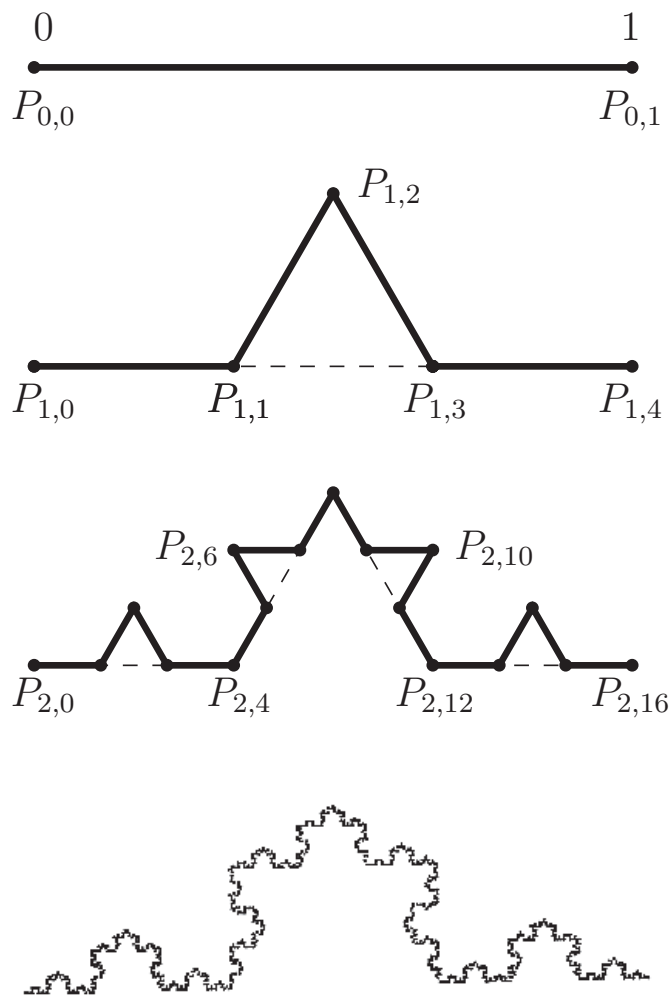

Figure 1

for balls in this metric space. Hence $\left(X, \varrho, \mu_{L}\right)$ is a $d$-space according to Definition 3.3 (ii). Furthermore,

$$
\begin{aligned}
\left|H\left(x_{k, l}\right)-H\left(x_{k, l-1}\right)\right| & =\left|P_{k, l}-P_{k, l-1}\right|=3^{-k}=4^{-k \varepsilon} \\
& =\varrho\left(x_{k, l}, x_{k, l-1}\right)
\end{aligned}
$$

where $l=1, \ldots, 4^{k}$, and by geometrical reasoning

$$
|H(x)-H(y)| \sim \varrho(x, y), \quad 0 \leq x \leq y \leq 1 .
$$

Hence,

$$
H:\left(X, \varrho, \mu_{L}\right) \quad \text { onto } \quad\left(\Gamma, \varrho_{2}, \mathcal{H}_{\Gamma}^{d}\right)
$$

with the Euclidean metric $\varrho_{2}$ in the plane $\mathbb{R}^{2}$ and the snowflake curve $\Gamma$, is a biLipschitzian map and an Euclidean chart of the metric space in (70) according to Definition 3.8. 
Remark 3.11. In other words, (71) illuminates in the special case (69) how the Euclidean chart

$$
H:\left(X_{1}, \varrho_{1}^{\varepsilon}, \mu_{L, 1}\right) \Longleftrightarrow\left(\Gamma, \varrho_{N}, \mathcal{H}_{\Gamma}^{d_{\varepsilon}}\right), \quad d_{\varepsilon}=1 / \varepsilon,
$$

according to Definition 3.8 looks like, where $X_{1}=[0,1]$ is the unit interval, furnished with the Euclidean metric $\varrho_{1}$, hence $\varrho_{1}(x, y)=|x-y|$, and the (one-dimensional) Lebesgue measure $\mu_{L, 1}$. In the above case one has $N=2$ and $\Gamma$ is the snowflake curve. For $0<\varepsilon \leq 1$ one gets by (72) compact $d_{\varepsilon}$-sets in $\mathbb{R}^{N}$ where necessarily $N \geq 1 / \varepsilon$

Example 3.12. Taking products of the maps (72) for different $\varepsilon$ 's one gets Euclidean charts of the closed unit cube $X_{n}=[0,1]^{n}$ equipped with the anisotropic quasi-metric

$$
\varrho_{a, n}(x, y)=|x-y|_{a} \quad \text { where } \quad|x|_{a} \sim \sup _{k}\left|x_{k}\right|^{1 / a_{k}}
$$

with respect to the anisotropy

$$
a=\left(a_{1}, \ldots, a_{n}\right), \quad 0<a_{1} \leq a_{2} \leq \cdots \leq a_{n}, \quad \sum_{k=1}^{n} a_{k}=n,
$$

and the $n$-dimensional Lebesgue measure $\mu_{L, n}$. Then

$$
\left(X_{n}, \varrho_{a, n}^{\varepsilon}, \mu_{L, n}\right)
$$

with

$$
\varepsilon=a_{1} \quad \text { and } \quad \varepsilon_{k}=a_{1} / a_{k} \quad \text { where } \quad k=1, \ldots, n,
$$

is a metric space (since $\varepsilon \leq 1$ and $\varepsilon_{k} \leq 1$ ) and might be considered as the snowflaked version of $\left(X_{n}, \varrho_{a, n}, \mu_{L, n}\right)$. In quite obvious notation one can represent (75) as the product

$$
\left(X_{n}, \varrho_{a, n}^{\varepsilon}, \mu_{L, n} t\right)=\prod_{k=1}^{n} \oplus\left(X_{1}, \varrho_{1}^{\varepsilon_{k}}, \mu_{L, 1}\right)
$$

of (one-dimensional) spaces in (72) with the (one-dimensional) bi-Lipschitzian maps

$$
H_{k}:\left(X_{1}, \varrho_{1}^{\varepsilon_{k}}, \mu_{L, 1}\right) \Longleftrightarrow\left(\Gamma^{k}, \varrho_{N_{k}}, \mathcal{H}_{\Gamma^{k}}^{d_{k}}\right) .
$$

Since $\varepsilon_{1}=1$ one may assume that $H_{1}$ is the identity. Otherwise $\Gamma^{k}$ are $d_{k}$-sets in some $\mathbb{R}^{N_{k}}$ with $d_{k}=1 / \varepsilon_{k}=a_{k} / a_{1}$. Let

$$
H=\left(H_{1}, \ldots, H_{n}\right), \quad d=\sum_{k=1}^{n} d_{k}, \quad N=\sum_{k=1}^{n} N_{k} .
$$

Then one gets the following assertion. 
Proposition 3.13. Let $X_{n}=[0,1]^{n}$ be the closed unit cube in $\mathbb{R}^{n}$. Let a and $\varrho_{a, n}$ be an anisotropy and the related quasi-metric as in (74) and (73). Then there is a bi-Lipschitzian map

$$
H:\left(X_{n}, \varrho_{a, n}^{a_{1}}, \mu_{L, n}\right) \Longleftrightarrow\left(\Gamma, \varrho_{N}, \mathcal{H}_{\Gamma}^{d}\right)
$$

of the indicated snowflaked version of $\left(X_{n}, \varrho_{a, n}, \mu_{L, n}\right)$ onto a compact $d$-set $\Gamma$ with $d=n / a_{1}$ in some $\mathbb{R}^{N}$.

Proof. Let $H$ be given by (78). Then $d=n / a_{1}$ follows from $d_{k}=a_{k} / a_{1}$ and (74). By (77) one gets (79) with

$$
\Gamma=\prod_{k=1}^{n} \oplus \Gamma^{k} \quad \text { being a } d \text {-set in } \mathbb{R}^{N},
$$

where $N$ is given by (78).

Example 3.14. We illustrate Proposition 3.13. Let $n=2$ and $X_{2}=[0,1]^{2}$. Let according to (76) and (74)

$$
\varepsilon_{2}=\frac{a_{1}}{a_{2}}=\frac{\log 3}{\log 4} \quad \text { and } \quad a_{1}\left(1+\frac{\log 4}{\log 3}\right)=2 .
$$

Then one can identify $H_{2}$ in (77) with $H$ in Example 3.10, the bi-Lipschitzian map of $[0,1]$ onto the snowflake curve, whereas $H_{1}$ is the identity. Hence in this case $H$ in (79) maps $X_{2}$ onto a cylindrical $d$-set in $\mathbb{R}^{3}$ with $d=1+\log 4 / \log 3$ (a number between 2 and 3 ) based on the snowflake curve in the plane cylindrical extended in the third direction in $\mathbb{R}^{3}$. In the general case each of the maps $H_{k}$ in (77) with $k \geq 2$ produces in some $\mathbb{R}^{N_{k}}$ a bizarre fractal curve which is a connected $d_{k}$-set. Then $\Gamma$ in (80) is the cartesian product of these wired curves producing a connected fractal surface in $\mathbb{R}^{N}$.

\section{Function spaces on $d$-spaces}

\subsection{Frames}

For $d$-spaces we abbreviate the Euclidean charts according to Definition 3.8 by $(X ; H)$ or, in case of doubt, by $(X, \varrho, \mu ; H)$ and call them $\varepsilon$-charts: One may also fix $\mu$ (or replace it by an equivalent measure) as the image measure

$$
\mu=\mathcal{H}_{\Gamma}^{d_{\varepsilon}} \circ H .
$$

This canonical choice of $\mu$ does not influence very much what follows now. However a more sophisticated analysis on $d$-spaces suggests to fix $\mu$ as in (81). We refer to Definition 5.3 and Theorem 5.5 below. As mentioned at the beginning of section 3.2 we always identify $\varrho$ with $\varrho$ in Theorem 3.1. In particular, $B(x, r)$ with $x \in X$ and $r>0$ are the balls in (61) with $\varrho$ in place of $\bar{\varrho}$. 
Proposition 4.1. Let $(X, \varrho, \mu)$ be a d-space. For any $k \in \mathbb{N}_{0}$ there are lattices and subordinated resolutions of unity

$$
\left\{x^{k, m}\right\}_{m=1}^{M_{k}} \subset X \quad \text { and } \quad\left\{\psi^{k, m}\right\}_{m=1}^{M_{k}}
$$

such that for some $c_{1}>0$ and $c_{2}>0$ (which are independent of $k$ )

$$
\begin{gathered}
\varrho\left(x^{k, m_{1}}, x^{k, m_{2}}\right) \geq c_{1} 2^{-k}, \quad k \in \mathbb{N}_{0}, \quad m_{1} \neq m_{2}, \\
X=\bigcup_{m=1}^{M_{k}} B_{k, m} \quad \text { with } \quad B_{k, m}=B\left(x^{k, m}, c_{2} 2^{-k}\right) \quad \text { for } k \in \mathbb{N}_{0},
\end{gathered}
$$

whereas $\psi^{k, m}$ are non-negative continuous functions on $X$ with

$$
\operatorname{supp} \psi^{k, m} \subset B_{k, m}, \quad k \in \mathbb{N}_{0}, \quad m=1, \ldots, M_{k},
$$

and

$$
\sum_{m=1}^{M_{k}} \psi^{k, m}(x)=1 \quad \text { where } \quad x \in X, \quad k \in \mathbb{N}_{0} .
$$

Proof. This follows from the corresponding assertions for $d$-sets in $\mathbb{R}^{n}$ as discussed at the beginning of section 2.2 and the existence of $\varepsilon$-charts.

Remark 4.2. Hence the above proposition is quite obvious if one relies on the existence of $\varepsilon$-charts. But the assertion itself is known on a larger scale and it is a cornerstone for the analysis on homogeneous spaces. One may consult [21] and the references given there. By the above arguments it follows that one may assume that

$$
M_{k} \sim 2^{d k} \quad \text { and } \quad \psi^{k, m} \in \operatorname{Lip}^{\varepsilon}(X), \quad k \in \mathbb{N}_{0},
$$

and $m=1, \ldots, M_{k}$. Here $\operatorname{Lip}^{\varepsilon}(X)$ is the Banach space of all complex continuous functions on $X$ such that

$$
\left\|f\left|\operatorname{Lip}^{\varepsilon}(X) \|=\sup _{x \in X}\right| f(x) \mid+\sup _{x \neq y} \frac{|f(x)-f(y)|}{\varrho^{\varepsilon}(x, y)}<\infty .\right.
$$

Recall that $0<\varepsilon<\varepsilon_{0}$, where $0<\varepsilon_{0} \leq 1$.

Let $\varepsilon-\Psi_{\Gamma}$ and $\varepsilon-\Psi_{\Gamma}^{s, p}$ be the frames introduced in Definition 2.11 for compact $d$ sets in $\mathbb{R}^{n}$. As has been mentioned at the end of Remark 2.15 to call them frames is justified by Theorem 2.14 (ii). We transfer these frames from $d_{\varepsilon}$-sets in some $\mathbb{R}^{n}$ to $d$-spaces. Let now

$$
\psi_{\beta}^{k, m}(\gamma)=\mathcal{H}_{\Gamma}^{d_{\varepsilon}}\left(B_{k, m}^{\Gamma}\right)^{-|\beta| / d_{\varepsilon}}\left(\gamma-\gamma^{k, m}\right)^{\beta} \psi^{k, m}(\gamma), \quad \gamma \in \Gamma,
$$

with $d_{\varepsilon}=d / \varepsilon$ and

$$
B_{k, m}^{\Gamma}=B\left(\gamma^{k, m}, 2^{-\varepsilon k}\right), \quad k \in \mathbb{N}_{0} ; \quad m=1, \ldots, M_{k},
$$


be the adapted version of $(19)$ with respect to $d_{\varepsilon}$-sets. We put

$$
\psi_{l}^{k, m}(x)=\left(\psi_{\beta}^{k, m} \circ H\right)(x)=\psi_{\beta}^{k, m}(H x), \quad x \in X,
$$

where $H$ is an $\varepsilon$-chart $(X ; H)$ according to Definition 3.8. In (90) we assume that $\gamma^{k, m}=H x^{k, m}$ and that there is an arbitrary one-to-one mapping $\beta \Leftrightarrow l$ of $\mathbb{N}_{0}^{n}$ onto $\mathbb{N}_{0}$ with $l=0$ if $\beta=0$. In particular we may put $\psi_{0}^{k, m}=\psi^{k, m}$ where $\psi^{k, m}$ has the same meaning as in (85), (86).

Definition 4.3. Let $(X, \varrho, \mu)$ be a $d$-space and let $(X ; H)$ be a respective $\varepsilon$-chart according to Definitions 3.3 and 3.8 .

(i) Let

$$
\varepsilon-\Psi_{\Gamma}=\left\{\psi_{\beta}^{k, m}: \beta \in \mathbb{N}_{0}^{n}, k \in \mathbb{N}_{0}, m=1, \ldots, M_{k}\right\}
$$

with (88) a corresponding $\varepsilon$-frame on the $d_{\varepsilon}$-set $\Gamma$. Then

$$
H-\Psi_{X}=\left(\varepsilon-\Psi_{\Gamma}\right) \circ H=\left\{\psi_{l}^{k, m}: l \in \mathbb{N}_{0}, k \in \mathbb{N}_{0}, m=1, \ldots, M_{k}\right\}
$$

with (90) is called an $H$-frame. Let $s>0$ and $1<p<\infty$. Then

$$
H-\Psi_{X}^{s, p}=\left\{(\mathrm{l}-\mathrm{qu})_{k, m}^{X}: l \in \mathbb{N}_{0}, k \in \mathbb{N}_{0}, m=1, \ldots, M_{k}\right\}
$$

with the $(s, p)$-l-quarks on $X$,

$$
(\mathrm{l}-\mathrm{qu})_{k, m}^{X}(x)=\mu\left(B_{k, m}\right)^{\frac{s}{d}-\frac{1}{p}} \psi_{l}^{k, m}(x), \quad x \in X,
$$

are the related $H$ - $(s, p)$-frames.

(ii) Let

$$
\nu=\left\{\nu_{k m}^{l} \in \mathbb{C}: l \in \mathbb{N}_{0}, k \in \mathbb{N}_{0}, m=1, \ldots, M_{k}\right\}
$$

and $1<p<\infty$. Then

$$
\ell_{p}^{X}=\left\{\nu:\left\|\nu \mid \ell_{p}^{X}\right\|=\left(\sum_{l, k, m}\left|\nu_{k m}^{l}\right|^{p}\right)^{1 / p}<\infty\right\} .
$$

Remark 4.4. This is the direct counterpart of Definition 2.11 for compact $d_{\varepsilon}$-sets. By (90), (23) and a suitable mapping $\beta \Leftrightarrow l$ one may assume that

$$
\left|\psi_{l}^{k, m}(x)\right| \leq c_{1} 2^{-\varepsilon c_{2} l}, \quad l \in \mathbb{N}_{0}, \quad k \in \mathbb{N}_{0}, \quad m=1, \ldots, M_{k},
$$

decays exponentially with respect to $l$, where $c_{1}$ and $c_{2}$ are suitable positive numbers which are independent of $l, k, m$, and $x$. Furthermore by (89) and the above mappings

$$
\mu\left(B_{k, m}\right)^{\frac{s}{d}-\frac{1}{p}} \sim \mathcal{H}_{\Gamma}^{d_{\varepsilon}}\left(B_{k m}^{\Gamma}\right)^{\frac{s}{\varepsilon} \cdot \frac{1}{d_{\varepsilon}}-\frac{1}{p}} .
$$

Hence, the $(s, p)$-l-quarks $(91)$ are the transferred $(s / \varepsilon, p)$ - $\beta$-quarks on the $d_{\varepsilon}$-set $\Gamma$ according to $(20)$. 
Remark 4.5. We summarize the procedure. Starting point is the quadruple $(X, \varrho, \mu ; H)$ consisting of the $d$-space $(X, \varrho, \mu)$ and the $\varepsilon$-chart $H$. The map

$$
H\left\{x^{k, m}\right\}=\left\{\gamma^{k, m}\right\} \subset \Gamma
$$

of an arbitrary admitted lattice according to (82)-(84) results in an admitted (approximate) lattice on the $d_{\varepsilon}$-set in some $\mathbb{R}^{n}$ where one may assume that it satisfies (13)-(14). Afterwards one transforms via $H^{-1}$ the $\varepsilon-\Psi_{\Gamma}$ frame and the $\varepsilon-\Psi^{s / \varepsilon, p}$ frame from the $d_{\varepsilon}$-set $\Gamma$ to $X$ including the normalizations.

\subsection{Spaces of positive smoothness}

According to Theorem 2.14 we have in case of $d$-sets in $\mathbb{R}^{n}$ intrinsic descriptions of the spaces $B_{p}^{s}(\Gamma)$ with $s>0$ and $1<p<\infty$ in terms of $\varepsilon-\Psi_{\Gamma}^{s, p}$ frames as introduced in Definition 2.11. Relying on section 4.1 one can extend these considerations to arbitrary $d$-spaces $(X, \varrho, \mu)$ or better to $\varepsilon$-charts $(X, \varrho, \mu ; H)$ of these $d$-spaces in a consistent way. If $1 \leq r<\infty$ then $L_{r}(X, \varrho, \mu)$ has the usual meaning normed by

$$
\left\|f \mid L_{r}(X, \varrho, \mu)\right\|=\left(\int_{X}|f(x)|^{r} \mu(\mathrm{d} x)\right)^{1 / r} .
$$

If one normalizes the measure $\mu$ for a given $\varepsilon$-chart $H$ according to $(81)$ then one gets the isometric map

$$
\left\|\left.f\left|L_{r}(X, \varrho, \mu) \|^{r}=\int_{\Gamma}\right|\left(f \circ H^{-1}\right)(\gamma)\right|^{r} \mathcal{H}_{\Gamma}^{d_{\varepsilon}}(\mathrm{d} \gamma) .\right.
$$

We need $L_{1}(X, \varrho, \mu)$ as a basic space in analogy to Theorem 2.14.

Definition 4.6. Let $(X, \varrho, \mu ; H)$ be an $\varepsilon$-chart of the $d$-space $(X, \varrho, \mu)$ according to the Definitions 3.3 and 3.8 . Let $s>0$ and $1<p<\infty$. Let the $H-(s, p)$-frames $H-\Psi_{X}^{s, p}$ and the sequence spaces $\ell_{p}^{X}$ be as introduced in Definition 4.3. Then $B_{p}^{s}(X, \varrho, \mu ; H)$, or $B_{p}^{s}(X ; H)$ for short, is the collection of all $f \in L_{1}(X, \varrho, \mu)$ which can be represented as

$$
f(x)=\sum_{l=0}^{\infty} \sum_{k=0}^{\infty} \sum_{m=1}^{M_{k}} \nu_{k m}^{l}(\mathrm{l} \text {-qu })_{k m}^{X}, \quad\left\|\nu \mid \ell_{p}^{X}\right\|<\infty,
$$

$x \in X$ (absolute convergence being in $\left.L_{1}(X, \varrho, \mu)\right)$. Furthermore,

$$
\left\|f\left|B_{p}^{s}(X ; H)\|=\inf \| \nu\right| \ell_{p}^{X}\right\|
$$

where the infimum is taken over all admissible representations (97).

Remark 4.7. This definition is the direct counterpart of the representation Theorem 2.14. In particular, the $(s, p)$-l-quarks $(\mathrm{l} \text {-qu })_{k m}^{X}$ originate from the $(s / \varepsilon, p)$ - $\beta$-quarks 
$(\beta \text {-qu })_{k m}^{\Gamma}$ including the normalizing factors as we mentioned explicitly in (94). Hence the absolute convergence in $L_{1}(X, \varrho, \mu)$ is not an additional assumption but a consequence of $\left\|\nu \mid \ell_{p}^{X}\right\|<\infty$. Furthermore this definition is consistent with what we know so far: If $(X, \varrho, \mu)$ is a $d$-set $\left(\Gamma, \varrho_{n}, \mathcal{H}_{\Gamma}^{d}\right)$ in some $\mathbb{R}^{n}$ and if $H=$ id is the identity then $B_{p}^{s}(X ; H)=B_{p}^{s}(\Gamma)$ according to Theorem 2.14 and the above definition. But otherwise one can introduce on $\left(\Gamma, \varrho_{n}, \mathcal{H}_{\Gamma}^{d} ; H\right)$ many scales of spaces $B_{p}^{s}(X ; H)$ which do not originate from trace spaces according to Definition 2.7. For example, a Lipschitz diffeomorphism $H$ of $\mathbb{R}^{n}$ onto itself preserves the property to be a $d$-set but destroys the differentiability properties (16) on which Definition 2.11 relies. Nevertheless as we shall see later on in this particular case all spaces $B_{p}^{s}(X ; H)$ coincide if $0<s<1$. But this is not the case if $s>1$ (one might think of Hölder-Zygmund spaces $\mathcal{C}^{s}=B_{\infty \infty}^{s}$ with $s>1$ on pieces of hyper-planes).

Theorem 4.8. Let $(X, \varrho, \mu ; H)$ be an $\varepsilon$-chart of the $d$-space $(X, \varrho, \mu)$ according to the Definitions 3.3 and 3.8. Let $B_{p}^{s}(X ; H)$ be the spaces introduced in Definition 4.6 where $s>0$ and $1<p<\infty$.

(i) Then $B_{p}^{s}(X ; H)$ is a Banach space. It is independent of all admissible $H$ - $(s, p)$ frames $H-\Psi_{X}^{s, p}$ according to Definition 4.3 and

$$
B_{p}^{s}(X, \varrho, \mu ; H)=B_{p}^{s / \varepsilon}\left(\Gamma, \varrho_{n}, \mathcal{H}_{\Gamma}^{d_{\varepsilon}}\right) \circ H
$$

where again $d_{\varepsilon}=d / \varepsilon$.

(ii) In addition let $s / \varepsilon \notin \mathbb{N}$. Then there is a linear and bounded map

$$
f \mapsto \nu(f)=\left\{\nu_{k m}^{l}(f)\right\}: B_{p}^{s}(X ; H) \mapsto \ell_{p}^{X}
$$

such that

$$
f(x)=\sum_{l, k, m} \nu_{k m}^{l}(f)(\mathrm{l}-\mathrm{qu})_{k m}^{X}, \quad x \in X
$$

with

$$
\left\|f\left|B_{p}^{s}(X ; H)\|\sim\| \nu(f)\right| \ell_{p}^{X}\right\|
$$

(equivalent norms where the equivalence constants are independent of $f$ ).

Proof. Here (98) means

$$
\left\|f \circ H\left|B_{p}^{s}(X ; H)\|\sim\| f\right| B_{p}^{s / \varepsilon}(\Gamma)\right\|, \quad f \in B_{p}^{s / \varepsilon}(\Gamma) .
$$

Otherwise part (i) follows from Theorem 2.14 (i), Definition 4.3, and the discussions in the Remarks 4.4, 4.5. Part (ii) is a consequence of Theorem 2.14 (ii) where also the curious restriction $s / \varepsilon \notin \mathbb{N}$ comes from. 


\subsection{Spaces of arbitrary smoothness}

We extend the scale of spaces $B_{p}^{s}(X ; H)$ according to Definition 4.6 to $s<0$ by duality. For this purpose we need some preparations.

Definition 4.9. Let $(X, \varrho, \mu ; H)$ be an $\varepsilon$-chart of the $d$-space $(X, \varrho, \mu)$ according to Definitions 3.3 and 3.8. Let $B_{p}^{s}(X ; H)$ be the spaces as introduced in Definition 4.6. Then

$$
D(X ; H)=\bigcap_{s>0} B_{p}^{s}(X ; H), \quad 1<p<\infty
$$

naturally equipped with a locally convex topology.

Remark 4.10. By well-known embedding theorems $D(X ; H)$ (including its locally convex topology) is independent of $p$. In particular, the topology of $D(X ; H)$ can be generated by countably many norms

$$
\left\|f \mid B_{2}^{s_{k}}(X ; H)\right\|, \quad k \in \mathbb{N}, \quad 0<s_{1}<s_{2}<\cdots s_{k} \rightarrow \infty
$$

if $k \rightarrow \infty$ of the Hilbert spaces $B_{2}^{s_{k}}(X ; H)$. By $(98)$ one can reduce these spaces to the Hilbert spaces $B_{2}^{\sigma_{k}}(\Gamma)$ with $\sigma_{k}=s_{k} / \varepsilon$ on a $d_{\varepsilon}$-set $\Gamma$ in some $\mathbb{R}^{n}$, which in turn are defined by $(12)$ as traces of the Hilbert spaces $B_{2}^{\sigma_{k}+\frac{n-d_{\varepsilon}}{2}}\left(\mathbb{R}^{n}\right)$. But in case of Hilbert spaces, $\operatorname{tr}_{\Gamma}$ in (12) is a retraction and there is a linear and bounded extension operator

$$
\operatorname{ext}_{\Gamma}: B_{2}^{\sigma_{k}}(\Gamma) \hookrightarrow B_{2}^{\sigma_{k}+\frac{n-d_{\varepsilon}}{2}}\left(\mathbb{R}^{n}\right)
$$

with

$$
\operatorname{tr}_{\Gamma} \circ \operatorname{ext}_{\Gamma}=\mathrm{id} \quad(\text { on } \Gamma) \text { and } \operatorname{supp}\left\{\operatorname{ext}_{\Gamma} g\right\} \subset B
$$

where $B$ is some (open) ball with $\Gamma \subset B$. We refer to Remark 2.10. Now it follows that the topology of $D(X ; H)$ can be reduced to the topology of $C^{\infty}(\bar{B})$ or $S\left(\mathbb{R}^{n}\right)$. Recall that both $C^{\infty}(\bar{B})$ and $S\left(\mathbb{R}^{n}\right)$ are nuclear locally convex spaces. We refer for details to [38, chapter 8], in particular sections 8.3.2, 8.3.3. Then $D(X ; H)$ is also a nuclear locally convex space. But we shall not need this assertion in the sequel.

Proposition 4.11. Let $(X, \varrho, \mu ; H)$ be an $\varepsilon$-chart of the $d$-space $(X, \varrho, \mu)$ according to Definitions 3.3 and 3.8, and let $D(X ; H)$ be the locally convex spaces as introduced in (99). Then $D(X ; H)$ is nuclear. Furthermore it is dense in all spaces $B_{p}^{s}(X ; H)$ with $s>0,1<p<\infty$, and all spaces $L_{r}(X, \varrho, \mu)$ with $1 \leq r<\infty$.

Proof. We justified in Remark 4.10 that $D(X ; H)$ is nuclear. The density of $D(X ; H)$ in $B_{p}^{s}(X ; H)$ follows immediately from Definition 4.6. The corresponding question for the spaces $L_{r}(X ; H)$ can be reduced by $(96)$ and $(99)$ to compact $d$-sets in $\mathbb{R}^{n}$. We have by the comments in Remark 4.10 that $D(\Gamma)=S\left(\mathbb{R}^{n}\right) \mid \Gamma$. This set is dense in $L_{r}(\Gamma)$. A short proof may be found in [41, pp. 6-7]. 
Remark 4.12. Let $D^{\prime}(X ; H)$ be the dual space of $D(X ; H)$ furnished with the usual (weak or strong) topology. Since $D(X ; H)$ is dense in $L_{r}(X)=L_{r}(X, \varrho, \mu)$ the standard dual pairing

$$
(f, g)=\int_{X} f(x) \cdot g(x) \mu(\mathrm{d} x), \quad f \in L_{r}(X), \quad g \in L_{r^{\prime}}(X),
$$

with $1 \leq r<\infty, \frac{1}{r}+\frac{1}{r^{\prime}}=1$, can be used to interpret $f \in L_{r}(X)$ as a distribution $f \in D^{\prime}(X ; H)$. As for the logical background of such an identification we first remark that $\mu$ is a Radon measure. This follows from (81) and the respective assertion for $\mathcal{H}_{\Gamma}^{d}$ which may be found in [41, Corollary 3.6, p. 6] (but it is a well known standard assertion). Then it follows from the Riesz representation theorem as stated in $[32$, Theorem 6.6, p. 97], that there is an one-to-one relation between $f \in L_{r}(X)$ and the complex Radon measure $f \mu \in D^{\prime}(X ; H)$. This justifies as usual,

$$
L_{r}(X) \hookrightarrow D^{\prime}(X ; H), \quad 1 \leq r<\infty .
$$

This inclusion can be extended to the continuous embeddings

$$
D(X ; H) \hookrightarrow B_{p}^{s}(X ; H) \hookrightarrow L_{p}(X) \hookrightarrow D^{\prime}(X ; H), \quad 1<p<\infty,
$$

$s>0$. The first (dense) embedding is covered by Proposition 4.11, the last one by (100) and the middle one can be reduced to (11), (12).

Definition 4.13. Let $(X, \varrho, \mu ; H)$ be an $\varepsilon$-chart of the $d$-space $(X, \varrho, \mu)$ according to the Definitions 3.3 and 3.8. Let $1<p<\infty$ and $s<0$. Then

$$
B_{p}^{s}(X ; H)=\left(B_{p^{\prime}}^{-s}(X ; H)\right)^{\prime}, \quad \frac{1}{p}+\frac{1}{p^{\prime}}=1,
$$

according to the dual pairing $\left(D(X ; H), D^{\prime}(X ; H)\right)$.

Remark 4.14. Here $B_{p^{\prime}}^{-s}(X ; H)$ are the spaces as introduced in Definition 4.6. Otherwise this definition is justified by the above preceding considerations. We refer also to [42, section 20.5 , pp. 208-209], where we dealt with constructions of this type in an $L_{2}$-setting.

Recall that $\mathbb{Z}$ is the collection of all integers and $\mathbb{N}_{0}$ is the collection of all nonnegative integers.

Theorem 4.15. Let $(X, \varrho, \mu ; H)$ be an $\varepsilon$-chart of the $d$-space $(X, \varrho, \mu)$ according to Definitions 3.3 and 3.8. Let $B_{p}^{s}(X ; H)$ with $1<p<\infty$ and $s \neq 0$ be the spaces as introduced in the Definitions 4.6 and 4.13.

(i) Then $B_{p}^{s}(X ; H)$ are Banach spaces,

$$
D(X ; H)=\bigcap_{s>0} B_{p}^{s}(X ; H) \quad \text { and } \quad D^{\prime}(X ; H)=\bigcup_{s<0} B_{p}^{s}(X ; H)
$$

where $D(X ; H)$ and $D^{\prime}(X ; H)$ have the same meaning as in $(99)$ and as at the beginning of Remark 4.12. 
(ii) Let, in addition $-s / \varepsilon \neq \mathbb{N}_{0}$. Then $D(X ; H)$ is dense in $B_{p}^{s}(X ; H)$.

(iii) Let, in addition, $s / \varepsilon \neq \mathbb{Z}$. Then $B_{p}^{s}(X ; H)$ is isomorphic to $\ell_{p}$.

Proof. Step 1. Part (i) with $s>0$ repeats simply Theorem 4.8 (i) and the definition (99) (for sake of completeness). If $s<0$, then $B_{p}^{s}(X ; H)$ is the dual of a Banach space and hence also a Banach space. The second part of (103) is now a consequence of these observations and standard arguments of dual spaces of locally convex spaces of the above type.

Step 2. We prove (iii). If $s>0$ then (98) reduces this problem to the spaces $B_{p}^{s / \varepsilon}(\Gamma)$ for the compact $d_{\varepsilon}$-set $\Gamma$ in some $\mathbb{R}^{n}$. Recall that the spaces $B_{p}^{\sigma}\left(\mathbb{R}^{n}\right)=$ $B_{p p}^{\sigma}\left(\mathbb{R}^{n}\right)$ with $\sigma \in \mathbb{R}$ and $1<p<\infty$ are isomorphic to $\ell_{p}$. We refer to [38, Theorem 2.11.2, p. 237]. If $0<s / \varepsilon \notin \mathbb{N}$ then it follows by Proposition 2.9 that $B_{p}^{s / \varepsilon}(\Gamma)$ is a complemented subspaces of $B_{p}^{\frac{s}{\varepsilon}+\frac{n-d_{\varepsilon}}{p}}\left(\mathbb{R}^{n}\right)$ and hence isomorphic to an infinitedimensional complemented subspace of $\ell_{p}$. But such a space is again isomorphic to $\ell_{p}$ (since $\ell_{p}$ is a prime Banach space). Details and references may be found in [38, section 2.11.1, p. 236]. The corresponding assertion for $s<0$ and $s / \varepsilon \notin \mathbb{Z}$ follows by duality.

Step 3. We prove (ii). The case $s>0$ is covered by Proposition 4.11. Let $s<0$. By (101) and duality one gets

$$
L_{p}(X) \hookrightarrow B_{p}^{s}(X ; H), \quad s<0, \quad 1<p<\infty .
$$

We claim that $L_{p}(X)$ is dense in $B_{p}^{s}(X ; H)$. If not, then one finds by the Hahn-Banach theorem a non-trivial functional $F$,

$$
F \in\left(B_{p}^{s}(X ; H)\right)^{\prime} \quad \text { with } \quad(F, g)=0 \text { for } g \in L_{p}(X) .
$$

By part (iii) and $-s / \varepsilon \notin \mathbb{N}_{0}$ it follows that $B_{p}^{s}(X ; H)$ is reflexive and one gets in particular

$$
(F, \varphi)=0 \quad \text { for } \quad F \in B_{p^{\prime}}^{-s}(X ; H) \quad \text { and } \quad \varphi \in D(X ; H) .
$$

Hence $F=0$ and one obtains a contradiction. As a consequence, (104) is a dense embedding. By Proposition 4.11 the set $D(X ; H)$ is dense in $L_{p}(X ; H)$. Then it is also dense in $B_{p}^{s}(X ; H)$.

Problem 4.16. Remove the nasty restrictions $-s / \varepsilon \notin \mathbb{N}_{0}$ and $s / \varepsilon \notin \mathbb{Z}$ in the above theorem.

Remark 4.17. So far we did not define spaces $B_{p}^{s}(X ; H)$ with $1<p<\infty$ and $s=0$. It comes out that this can be done by real interpolation

$$
B_{p}^{0}(X ; H)=\left(B_{p}^{s}(X ; H), B_{p}^{-s}(X ; H)\right)_{1 / 2, p}, \quad 0<s<\varepsilon,
$$

where the interpolation spaces are independent of $s$ and even of the $\varepsilon$-charts $H$, hence

$$
B_{p}^{0}(X)=\left(B_{p}^{s}(X), B_{p}^{-s}(X)\right)_{1 / 2, p}
$$


in the notation of the spaces introduced in the next section 4.4. Then the interpolation formula (105) is a special case of [49, Theorem 3.2].

\subsection{The standard approach}

The above approach to function spaces on $d$-spaces according to Definition 3.3 rests on two ingredients: An elaborated theory of corresponding spaces on compact $d$ sets in $\mathbb{R}^{n}$ as described in section 2 on the one hand and the possibility to transfer this theory via Euclidean charts according to Definition 3.8 to (abstract) $d$-spaces on the other hand. As a result one gets families of spaces $B_{p}^{s}(X, \varrho, \mu ; H)$ with $s \in \mathbb{R}$, $1<p<\infty$, for $\varepsilon$-charts $(X, \varrho, \mu ; H)$ of the $d$-spaces $(X, \varrho, \mu)$ as treated in the preceding sections $4.2,4.3$. This might be interpreted as a new approach to the question,

$$
\text { How to measure smoothness on }(X, \varrho, \mu) \text {, }
$$

where in general $X$ is a set, $\varrho$ a quasi-metric and $\mu$ a (regular) Borel measure. Problems of type (106) attracted a lot of attention in the last decade and especially in very recent times. First we complement now corresponding comments and the literature given in Remark 3.5 by some more specific references dealing with function spaces.

Based on [28], R. S. Strichartz developed in [37] a theory of Hölder-Zygmund, Besov and Sobolev spaces of higher order on the Sierpinski gasket and other post critical finite self-similar fractals. In general, these spaces are different from the trace spaces according to Definition 2.7 which we considered in detail in section 2. Since a long time it is well known that classical Besov spaces and (fractional) Sobolev spaces on $\mathbb{R}^{n}$ are closely connected with Gauss-Weierstrass semi-groups, CauchyPoisson semi-groups and the underlying Bessel potentials and Riesz potentials. We refer to $[38$, sections 2.5.2, 2.5.3] for details and to [40, section 1.8.1] for a short description. There one finds also the necessary references. It is quite natural to ask for fractal counterparts. This works to some extent and under some restrictions $(d$-sets or Sierpinski gasket). Details and further references may be found in [24,29,52,53]. As far as Sobolev spaces on metric spaces are concerned we refer for different approaches to $[16,30]$.

After having complemented the literature given in Remark 3.5 we come now to the description of the most elaborated method to handle function spaces on quasimetric spaces, which we call the standard approach and which is not covered by the above references. We restrict ourselves to the simplest case and follow partly the presentation given in [46]. In particular we always assume that $(X, \varrho, \mu)$ is a $d$ set according to Definition 3.3 (ii), although what follows applies equally to general spaces of homogeneous type as introduced in Definition 3.3 (i) (after some technical modifications). We always assume that $\varepsilon_{0}$ has the same meaning as in Theorem 3.1 and we identify $\varrho$ with $\varrho$ resulting in (60) with $\varrho$ in place of $\varrho$ where $0<\varepsilon \leq \varepsilon_{0}$. Let

$$
L_{r}(X)=L_{r}(X, \varrho, \mu), 1 \leq r<\infty, \quad \text { and } \quad \operatorname{Lip}^{\varepsilon}(X), 0<\varepsilon<\varepsilon_{0}
$$


be the same Banach spaces as above, normed by (95) and (87), respectively. Then $\operatorname{Lip}^{\varepsilon}(X)$ is dense in $L_{2}(X)$. We refer to $[9,17]$. Hence the dual pairing

$$
\left(\operatorname{Lip}^{\varepsilon}(X),\left(\operatorname{Lip}^{\varepsilon}(X)\right)^{\prime}\right) \text { with }\left(L_{2}(X)\right)^{\prime}=L_{2}(X),
$$

as the usual identification makes sense. As for the logical background we refer to the discussion in Remark 4.12. It is well known that all spaces $B_{p q}^{s}\left(\mathbb{R}^{n}\right)$ and $F_{p q}^{s}\left(\mathbb{R}^{n}\right)$ (which will not be defined here in detail) can be characterized in terms of so-called local means within the dual pairing $\left(S\left(\mathbb{R}^{n}\right), S^{\prime}\left(\mathbb{R}^{n}\right)\right)$. One may consult $[40$, sections $1.8 .4,2.4 .6,2.5 .3]$. It is remarkable that there is a partial substitute under the above assumptions which goes back to [9]. We rely here on the modification according to [17] adapted to our situation. There are a sequence of complex-valued function

$$
S_{m}(x, y) \quad \text { with } x \in X, y \in X \text {, and } m \in \mathbb{N}_{0}
$$

and a number $C>0$ with the following properties:

$$
\begin{gathered}
S_{m}(x, y)=0 \text { if } \varrho(x, y) \geq C 2^{-m} \text { and }\left|S_{m}(x, y)\right| \leq C 2^{d m}, \\
\left\{\begin{array}{l}
\left|S_{m}(x, y)-S_{m}\left(x^{\prime}, y\right)\right| \leq C 2^{m(d+\varepsilon)} \varrho^{\varepsilon}\left(x, x^{\prime}\right), \\
\left|S_{m}(x, y)-S_{m}\left(x, y^{\prime}\right)\right| \leq C 2^{m(d+\varepsilon)} \varrho^{\varepsilon}\left(y, y^{\prime}\right),
\end{array}\right. \\
\left|S_{m}(x, y)-S_{m}\left(x^{\prime}, y\right)-S_{m}\left(x, y^{\prime}\right)+S_{m}\left(y, y^{\prime}\right)\right| \\
\leq C 2^{m(d+2 \varepsilon)} \varrho^{\varepsilon}\left(x, x^{\prime}\right) \varrho^{\varepsilon}\left(y, y^{\prime}\right)
\end{gathered}
$$

for all $x \in X, x^{\prime} \in X, y \in X, y^{\prime} \in X$ and

$$
\int_{X} S_{m}(x, y) \mu(\mathrm{d} y)=\int_{X} S_{m}(x, y) \mu(\mathrm{d} x)=1 .
$$

Let

$$
E_{0}(x, y)=S_{0}(x, y) \quad \text { and } \quad E_{m}(x, y)=S_{m}(x, y)-S_{m-1}(x, y)
$$

if $m \in \mathbb{N}$. Then we have

$$
\int_{X} E_{m}(x, y) \mu(\mathrm{d} y)=\int_{X} E_{m}(x, y) \mu(\mathrm{d} x)=0 \quad \text { for } \quad m \in \mathbb{N} .
$$

Now $E_{l}$ given by

$$
\left(E_{l} f\right)(x)=\int_{X} E_{l}(x, y) f(y) \mu(\mathrm{d} y) \quad \text { with } \quad l \in \mathbb{N}_{0},
$$

are the local means we are looking for.

Definition 4.18. Let $d>0$ and let $(X, \varrho, \mu)$ be a $d$-space according to Definition 3.3 equipped with the quasi-metric $\varrho$ (identified with $\varrho$ in $(60)$ ) where $0<\varepsilon=\varepsilon_{0} \leq 1$ 
has the same meaning as there. Let $1<p<\infty$ and $-\varepsilon<s<\varepsilon$. Let $E_{l} f$ be the local means according to (108). Then $B_{p}^{s}(X, \varrho, \mu)=B_{p}^{s}(X)$ is the collection of all $f \in\left(\operatorname{Lip}^{\varepsilon}(X)\right)^{\prime}$ such that

$$
\left\|f \mid B_{p}^{s}(X)\right\|=\left(\sum_{j=0}^{\infty} 2^{j s p}\left\|E_{j} f \mid L_{p}(X)\right\|^{p}\right)^{1 / p}<\infty .
$$

Remark 4.19. The definition is justified by the dual pairing (107). These spaces are Banach spaces which are independent of the chosen local means. Similarly one can introduce (inhomogeneous) spaces $B_{p q}^{s}(X)$ and $F_{p q}^{s}(X)$ with $1<p<\infty, 1 \leq q \leq \infty$ and $-\varepsilon<s<\varepsilon$. Furthermore there are several extensions of (homogeneous and nonhomogeneous) spaces of $B$-type and $F$-type on more general spaces of homogeneous type according to Definition 3.3 (i) and to some values $p=\infty, p \leq 1$, and $q \leq 1$. However the admitted smoothness $s$ of this direct approach is always limited by $|s|<\varepsilon$. Spaces of this type have a rather substantial history covering in particular the above case. The analysis on spaces of homogeneous type started with [8]. As far as the above function spaces are concerned we refer here to the surveys [20,21], the contributions $[17,18]$, and the recent papers $[19,22,46,49-51]$, reflecting at least partly some recent developments.

\subsection{Spaces of restricted smoothness}

So far we have the spaces $B_{p}^{s}(X, \varrho, \mu ; H)=B_{p}^{s}(X ; H)$ with $1<p<\infty, s \in \mathbb{R}$, $s \neq 0$ as introduced in the Definitions 4.6, 4.13 on the one hand, and the spaces $B_{p}^{s}(X, \varrho, \mu)=B_{p}^{s}(X)$ according to Definition 4.18 with $1<p<\infty,-\varepsilon<s<\varepsilon$, on the other hand. We wish to prove that these spaces coincide for given $p$ with $1<p<\infty$ and admitted $s$. For this purpose we transfer the atomic representation Theorem 2.23 from $d$-sets to $d$-spaces.

Definition 4.20. Let $(X, \varrho, \mu)$ be a $d$-space according to Definition 3.3 where the quasi-metric $\varrho$ satisfies $(60)$ with $\varepsilon_{0}$ (and $\bar{\varrho}=\varrho$ ). Let $B_{k, m}$ be the same balls as in (84). Let $0<\varepsilon<\varepsilon_{0}$ and let $\operatorname{Lip}^{\varepsilon}(X)$ be the above Banach spaces normed by (87). Let $0<s<\varepsilon$. Then $a_{X}^{k, m} \in \operatorname{Lip}^{\varepsilon}(X)$ is called an $(s, p)$ - $\varepsilon$-atom if for $k \in \mathbb{N}_{0}$ and $m=1, \ldots, M_{k}$,

$$
\begin{gathered}
\operatorname{supp} a_{X}^{k, m} \subset B_{k, m}, \\
\left|a_{X}^{k, m}(x)\right| \leq \mu\left(B_{k, m}\right)^{\frac{s}{d}-\frac{1}{p}}, \quad x \in X,
\end{gathered}
$$

and

$$
\left|a_{X}^{k, m}(x)-a_{X}^{k, m}(y)\right| \leq \mu\left(B_{k, m}\right)^{\frac{s}{d}-\frac{1}{p}-\frac{\varepsilon}{d}} \varrho^{\varepsilon}(x, y)
$$

with $x \in X, y \in X$. 
Remark 4.21. This is the direct counterpart of Definition 2.20 with respect to the metric $\varrho^{\varepsilon}$ (we indicated $\varepsilon$ although it has an other meaning as for the $(s, p)^{*}$ - $\varepsilon$-atoms in Definition 2.20). On the other hand, the $(s, p)$-l-quarks (l-qu) $)_{k, m}^{X}$ in (91) are special atoms of the above type (ignoring irrelevant constants). This follows from the reduction of these functions via (90) to the functions in (19) and with (16) (for $|\alpha|=1$ ): By the mean value theorem and (66) one gets for $x \in X, y \in X$,

$$
\left|(\mathrm{l}-\mathrm{qu})_{k, m}^{X}(x)-(\mathrm{l}-\mathrm{qu})_{k, m}^{X}(y)\right| \leq c \mu\left(B_{k, m}\right)^{\frac{s}{d}-\frac{1}{p}-\frac{\varepsilon}{d}} \varrho^{\varepsilon}(x, y)
$$

where $c>0$ is an unimportant universal constant. In particular, the right-hand sides of (109), (110) are the correct normalizing factors. This follows from transferring (36), (37) with $s / \varepsilon$ and the $d / \varepsilon$-set $\Gamma$ in place of $s$ and the $d$-set $\Gamma$ to the $d$-space $(X, \varrho, \mu)$. The obvious counterpart of the sequence spaces $(32),(33)$ and $(92),(93)$ is given by

$$
\nu=\left\{\nu_{k m} \in \mathbb{C}: k \in \mathbb{N}_{0}, m=1, \ldots, M_{k}\right\}
$$

and

$$
\ell_{p}^{X, 0}=\left\{\nu:\left\|\nu \mid \ell_{p}^{X, 0}\right\|=\left(\sum_{k=0}^{\infty} \sum_{m=1}^{M_{k}}\left|\nu_{k m}\right|^{p}\right)^{1 / p}<\infty\right\}
$$

where $1<p<\infty$.

Theorem 4.22. Let $(X, \varrho, \mu ; H)$ be an $\varepsilon$-chart of the $d$-space $(X, \varrho, \mu)$ according to the Definitions 3.3, 4.20, 3.8. Let $1<p<\infty$ and $0<\varepsilon<\varepsilon_{0}$. Let $B_{p}^{s}(X ; H)$ as introduced in the Definitions 4.6, 4.13 and let $B_{p}^{s}(X)$ be the spaces according to Definition 4.18 .

(i) Let $-\varepsilon<s<\varepsilon$ and $s \neq 0$. Then

$$
B_{p}^{s}(X ; H)=B_{p}^{s}(X)
$$

(ii) Let $0<s<\varepsilon$. Then $B_{p}^{s}(X)$ is the collection of all $f \in L_{1}(X)$ which can be represented as

$$
f(x)=\sum_{k=0}^{\infty} \sum_{m=1}^{M_{k}} \nu_{k m} a_{X}^{k, m}(x), \quad x \in X, \quad\left\|\nu \mid \ell_{p}^{X, 0}\right\|<\infty
$$

where $a_{X}^{k, m}$ are the $(s, p)$ - $\varepsilon$-atoms on $X$ according to Definition 4.20. Furthermore,

$$
\left\|f\left|B_{p}^{s}(X)\|\sim \inf \| \nu\right| \ell_{p}^{X, 0}\right\|
$$

where the infimum is taken over all admissible representations (112). 
Proof. Step 1. Let $0<s<\varepsilon$. Then we have (98). Furthermore by the corresponding comments in Remark 4.21 the transferred $(s / \varepsilon, p)^{*}$ - $\varepsilon$-atoms for the $d / \varepsilon$-set $\Gamma$ according to Definition 2.20 are the above $(s, p)$ - $\varepsilon$-atoms for $X$ including the normalizations. Then Theorem 2.23 proves the representation (112) and the equivalence (113). However this coincides with the atomic representation of the spaces $B_{p}^{s}(X)$ according to [21, Theorem 1.1]. We refer also to [50, Lemma 2.3]. Then one gets the desired assertion for $0<s<\varepsilon$.

Step 2. Let $-\varepsilon<s<0$. It follows from [21, Lemma 1.8, p. 18], that

$$
\left(B_{p}^{s}(X)\right)^{\prime}=B_{p^{\prime}}^{-s}(X), \quad 1<p<\infty, \quad \frac{1}{p}+\frac{1}{p^{\prime}}=1
$$

Together with (102) one gets (111) for $-\varepsilon<s<0$.

Remark 4.23. The above theorem combines two largely independent assertions. First we proved that for given $p$ and $s$ with $1<p<\infty$ and $-\varepsilon<s<\varepsilon, s \neq 0$, the spaces $B_{p}^{s}(X ; H)$ are the same for all admitted $\varepsilon$-charts and that one has for $0<s<\varepsilon$ the atomic representation (112). Secondly we identified these spaces with the spaces $B_{p}^{s}(X)$ according to Definition 4.18 using corresponding assertions for these spaces proved in $[21,50]$ (and in the papers quoted there). Let the $d$-space $X=\Gamma$ be a $d$-set in $\mathbb{R}^{n}$ and let $B_{p}^{s}(\Gamma)$ and $B_{p}^{s}(X)$ be the corresponding spaces as introduced in the Definitions 2.7 and 4.18 , respectively. Then one gets

$$
B_{p}^{s}(\Gamma)=B_{p}^{s}(X), \quad 0<s<1, \quad 1<p<\infty,
$$

as a special case of the above theorem. This assertion, in the larger context of $B_{p q}^{s}$ spaces, is the main result of [50]. The proof given there is based on atomic representations for the spaces $B_{p q}^{s}(X)$ and quarkonial decompositions for the spaces $B_{p q}^{s}(\Gamma)$ as in Theorem 2.14.

\section{Applications}

It is not our aim to deal systematically with applications. We sketch two examples.

\subsection{Entropy numbers}

Let $A$ and $B$ be two Banach spaces, let $U_{A}$ be the unit ball in $A$, and let $T: A \hookrightarrow B$ be linear and continuous. Then for all $k \in \mathbb{N}$ the $k$ th entropy number $e_{k}(T)$ of $T$ is defined as the infimum of all $\delta>0$ such that $T\left(U_{A}\right)$ can be covered by $2^{k-1}$ balls of radius $\delta$ in $B$.

Let $(X, \varrho, \mu ; H)$ be an $\varepsilon$-chart of the $d$-space $(X, \varrho, \mu)$ according to the Definitions 3.3 and 3.8. Let $B_{p}^{s}(X ; H)$ with $s>0$ and $1<p<\infty$ be the spaces introduced in Definition 4.6. Recall that $a_{k} \sim b_{k}$ for two sequences of positive numbers means that

$$
c_{1} a_{k} \leq b_{k} \leq c_{2} a_{k} \quad \text { for some } 0<c_{1} \leq c_{2}<\infty \text { and all } k \in \mathbb{N} .
$$


Theorem 5.1. Let

$$
0<s_{2}<s_{1}<\infty, \quad 1<p_{1}<\infty, \quad 1<p_{2}<\infty,
$$

and

$$
s_{1}-d / p_{1}>s_{2}-d / p_{2}
$$

Then the embedding

$$
\mathrm{id}^{X}: B_{p_{1}}^{s_{1}}(X ; H) \hookrightarrow B_{p_{2}}^{s_{2}}(X ; H)
$$

is compact and

$$
e_{k}\left(\mathrm{id}^{X}\right) \sim k^{-\frac{s_{1}-s_{2}}{d}} \quad \text { for } \quad k \in \mathbb{N} \text {. }
$$

Proof. By (98)

$$
H: f \mapsto f \circ H: B_{p}^{s / \varepsilon}\left(\Gamma, \varrho_{n}, \mathcal{H}_{\Gamma}^{d_{\varepsilon}}\right) \hookrightarrow B_{p}^{s}(X, \varrho, \mu ; H)
$$

is an isomorphic map where $\Gamma$ is a $d / \varepsilon$-set in some $\mathbb{R}^{n}$. Then

$$
\operatorname{id}^{X}=H \circ \operatorname{id}^{\Gamma} \circ H^{-1}
$$

with

$$
\operatorname{id}^{\Gamma}: B_{p_{1}}^{s_{1} / \varepsilon}(\Gamma) \hookrightarrow B_{p_{2}}^{s_{2} / \varepsilon}(\Gamma)
$$

Hence,

$$
e_{k}\left(\operatorname{id}^{X}\right) \sim e_{k}\left(\operatorname{id}^{\Gamma}\right) \sim k^{-\frac{s_{1}-s_{2}}{d}}, \quad k \in \mathbb{N},
$$

where the latter equivalence is covered by [41, Theorem 20.6, p. 166] (here $\varepsilon$ cancels out since $\Gamma$ is a $d / \varepsilon$-set).

Remark 5.2. Entropy numbers play a crucial role in the spectral theory of diverse operators. Detail may be found in $[11,41,42]$.

\subsection{Riesz potentials}

Let $\Gamma=\left(\Gamma, \varrho_{n}, \mathcal{H}_{\Gamma}^{d}\right)$ be a compact $d$-set in $\mathbb{R}^{n}$ with $0<d<n$ according to Definition 2.3 and let $I_{\varkappa}^{\Gamma}$,

$$
\left(I_{\varkappa}^{\Gamma} f\right)(\gamma)=\int_{\Gamma} \frac{f(\delta)}{|\gamma-\delta|^{d-\varkappa}} \mathcal{H}_{\Gamma}^{d}(\mathrm{~d} \delta), \quad 0<\varkappa<d,
$$

$\gamma \in \Gamma$, be Riesz potentials on $\Gamma$. These operators have been studied in detail in $[52,53]$. It comes out, in particular, that $I_{\varkappa}^{\Gamma}$ is a positive self-adjoint compact operator in $L_{2}(\Gamma)$. Let

$$
\lambda_{1} \geq \lambda_{2} \geq \cdots \geq \lambda_{k} \geq \cdots \rightarrow 0 \quad \text { if } \quad k \rightarrow \infty,
$$

be the positive eigenvalues of $I_{\varkappa}^{\Gamma}$ repeated according to multiplicity and ordered by magnitude. Then

$$
\lambda_{k} \sim k^{-\varkappa / d}, \quad k \in \mathbb{N} \text {. }
$$


We refer for details and proofs to [52, Theorem 3.3, Corollary 3.4] and [53]. We developed in [46] a corresponding theory for $d$-spaces $X=(X, \varrho, \mu)$ according to Definition 3.3. The outcome is the following: For any $\varkappa$ with $0<\varkappa<d$ there is an equivalent quasi-metric $\varrho_{\varkappa}, \varrho \sim \varrho_{\varkappa}$, such that the Riesz potential $I_{\varkappa}^{X}$,

$$
\left(I_{\varkappa}^{X} f\right)(x)=\int_{X} \frac{f(y)}{\varrho_{\varkappa}^{d-\varkappa}(x, y)} \mu(\mathrm{d} y), \quad x \in X,
$$

is a non-negative self-adjoint compact operator in $L_{2}(X)$ with $(115)$, (116) for its positive eigenvalues. All proofs in [52] and [46] are based on the theory of the spaces $B_{p}^{s}$ (and some generalizations). To which extent one has to replace $\varrho$ by an equivalent quasi-metric $\varrho_{\varkappa}$ is not so clear. But even in the Euclidean case (114) it seems to be doubtful whether one can replace the Euclidean metric $|\gamma-\delta|$ by an arbitrary equivalent quasi-metric $\varrho(\gamma, \delta)$ (which might be even non-continuous) if one wishes to get the above assertions. We refer for a corresponding discussion to [46, Remark 3.8]. The proofs in [46] are direct (long and complicated). We mentioned there Euclidean charts as a future tool but we did not use it (we inserted this idea at the very last moment when the rest of the paper was finished). But now we describe how one can reduce the operators in (117) to corresponding operators of type (114). The problem of equivalent quasi-metrics $\varrho_{\varkappa} \sim \varrho$ disappears by introducing a distinguished class of $d$-spaces which seems to be especially well adapted for a substantial analysis on it.

Definition 5.3. A $d$-space $(X, \varrho, \mu)$ as introduced in Definition 3.3 is called regular if there exists an $\varepsilon$-chart according to Definition 3.8 such that

$$
\mu=\mathcal{H}_{\Gamma}^{d_{\varepsilon}} \circ H \quad \text { and } \quad|H(x)-H(y)|=\varrho^{\varepsilon}(x, y), \quad x \in X, \quad y \in X .
$$

Remark 5.4. In other words, if $(X, \varrho, \mu ; H)$ is an $\varepsilon$-chart according to Definition 3.8 then we replace (if necessary) the original measure and the original quasi-metric by equivalent ones in (118). We refer for justification to (81), (66) and Theorem 3.1.

Theorem 5.5. Let $X=(X, \varrho, \mu)$ be a regular d-space according to Definition 5.3 and let $0<\varkappa<d$. Then the Riesz potential $I_{\varkappa}^{X}$,

$$
\left(I_{\varkappa}^{X} f\right)(x)=\int_{X} \frac{f(y)}{\varrho^{d-\varkappa}(x, y)} \mu(\mathrm{d} y), \quad x \in X,
$$

is a positive self-adjoint compact operator in $L_{2}(X)$ with (115), (116) for its positive eigenvalues.

Proof. We adapt (114) to (67), hence,

$$
\left(I_{\varkappa / \varepsilon}^{\Gamma} f\right)(\gamma)=\int_{\Gamma} \frac{f(\delta)}{|\gamma-\delta|^{\frac{d-\varkappa}{\varepsilon}}} \mathcal{H}_{\Gamma}^{d_{\varepsilon}}(\mathrm{d} \delta), \quad \gamma \in \Gamma,
$$


where $\Gamma$ is a $d / \varepsilon$-set in some $\mathbb{R}^{n}$. By (118) and (96) it follows that $H$ and $H^{-1}$ generate unitary mappings between $L_{2}(\Gamma)$ and $L_{2}(X)$ (denoted again by $H$ and $H^{-1}$ ). We claim that

$$
I_{\varkappa}^{X}=H \circ I_{\varkappa / \varepsilon}^{\Gamma} \circ H^{-1}
$$

are unitary equivalent, where $I_{\varkappa}^{X}$ and $I_{\varkappa / \varepsilon}^{\Gamma}$ are given by (119) and (120), respectively. We calculate the right-hand side of (121) for, say, $f \in L_{2}(X)$. Then

$$
\left(H \circ I_{\varkappa / \varepsilon}^{\Gamma} \circ H^{-1} f\right)(x)=\int_{\Gamma} \frac{f\left(H^{-1} \delta\right)}{|H x-\delta|^{\frac{d-\varkappa}{\varepsilon}}} \mathcal{H}_{\Gamma}^{d_{\varepsilon}}(\mathrm{d} \delta) .
$$

We transform the integral from $\Gamma$ to $X$ by $\delta=H y$. Using the two normalizations in (118) it follows that the right-hand side of (122) equals

$$
\int_{X} \frac{f(y)}{|H x-H y|^{\frac{d-\varkappa}{\varepsilon}}} \mu(\mathrm{d} y)=\int_{X} \frac{f(y)}{\varrho(x, y)^{d-\varkappa}} \mu(\mathrm{d} y) .
$$

This proves (121). Hence, $I_{\varkappa}^{X}$ is unitary equivalent to $I_{\varkappa / \varepsilon}^{\Gamma}$ where $\Gamma$ is a $d / \varepsilon$-set. Now the theorem follows from [52, Theorem 3.3, Corollary 3.4], as described in (114)-(116) applied to $I_{\varkappa / \varepsilon}^{\Gamma}$.

Remark 5.6. Compared with (117) one has now the distinguished equivalent quasinorm which originates from (118). In [46] we introduced classes of equivalent quasinorms on $(X, \varrho, \mu)$ asking for distinguished representatives. But the notion of regular $d$-spaces according to Definition 5.3 seems to be the better choice. Maybe Theorem 5.5 is the first step to a substantial analysis on regular $d$-spaces.

\section{References}

[1] D. R. Adams and L. I. Hedberg, Function spaces and potential theory, Grundlehren der Mathematischen Wissenschaften, vol. 314, Springer-Verlag, Berlin, 1996.

[2] P. Assouad, Étude d'une dimension métrique liée à la possibilité de plongements dans $\mathbf{R}^{n}$, C. R. Acad. Sci. Paris Sér. A-B 288 (1979), no. 15, A731-A734.

[3] , Plongements lipschitziens dans $\mathbf{R}^{n}$, Bull. Soc. Math. France 111 (1983), no. 4, 429448

[4] M. Bricchi, Existence and properties of h-sets, Georgian Math. J. 9 (2002), no. 1, 13-32.

[5] - Complements and results on h-sets, Function spaces, differential operators and nonlinear analysis (Teistungen, 2001), 2003, pp. 219-229.

[6] Tailored Besov spaces and h-sets, Math. Nachr. 263/264 (2004), 36-52.

[7] M. Christ, Lectures on singular integral operators, CBMS Regional Conference Series in Mathematics, vol. 77, Published for the Conference Board of the Mathematical Sciences, Washington, DC, 1990.

[8] R. R. Coifman and G. Weiss, Analyse harmonique non-commutative sur certains espaces homogènes, Springer-Verlag, Berlin, 1971. 
[9] G. David, J.-L. Journé, and S. Semmes, Opérateurs de Calderón-Zygmund, fonctions paraaccrétives et interpolation, Rev. Mat. Iberoamericana 1 (1985), no. 4, 1-56.

[10] G. David and S. Semmes, Fractured fractals and broken dreams, Oxford Lecture Series in Mathematics and its Applications, vol. 7, The Clarendon Press Oxford University Press, New York, 1997.

[11] D. E. Edmunds and H. Triebel, Function spaces, entropy numbers, differential operators, Cambridge Tracts in Mathematics, vol. 120, Cambridge University Press, Cambridge, 1996.

[12] K. J. Falconer, The geometry of fractal sets, Cambridge Tracts in Mathematics, vol. 85, Cambridge University Press, Cambridge, 1986.

[13] M. Frazier and B. Jawerth, Decomposition of Besov spaces, Indiana Univ. Math. J. 34 (1985), no. $4,777-799$

[14] _ A discrete transform and decompositions of distribution spaces, J. Funct. Anal. 93 (1990), no. $1,34-170$

[15] M. Frazier, B. Jawerth, and G. Weiss, Littlewood-Paley theory and the study of function spaces, CBMS Regional Conference Series in Mathematics, vol. 79, Published for the Conference Board of the Mathematical Sciences, Washington, DC, 1991

[16] P. Hajłasz and P. Koskela, Sobolev met Poincaré, Mem. Amer. Math. Soc. 145 (2000), no. 688, $1-101$.

[17] Y. Han, Inhomogeneous Calderón reproducing formula on spaces of homogeneous type, J. Geom. Anal. 7 (1997), no. 2, 259-284.

[18] Y. Han, S. Lu, and D. Yang, Inhomogeneous Besov and Triebel-Lizorkin spaces on spaces of homogeneous type, Approx. Theory Appl. (N.S.) 15 (1999), no. 3, 37-65.

[19] - Inhomogeneous discrete Calderón reproducing formulas for spaces of homogeneous type, J. Fourier Anal. Appl. 7 (2001), no. 6, 571-600.

[20] Y. S. Han and E. T. Sawyer, Littlewood-Paley theory on spaces of homogeneous type and the classical function spaces, Mem. Amer. Math. Soc. 110 (1994), no. 530.

[21] Y. Han and D. Yang, New characterizations and applications of inhomogeneous Besov and Triebel-Lizorkin spaces on homogeneous type spaces and fractals, Dissertationes Math. (Rozprawy Mat.) 403 (2002), 102.

[22] _ Some new spaces of Besov and Triebel-Lizorkin type on homogeneous spaces, Studia Math. 156 (2003), no. 1, 67-97.

[23] J. Heinonen, Lectures on analysis on metric spaces, Universitext, Springer-Verlag, New York, 2001.

[24] J. Hu and M. Zähle, Potential spaces on fractals, Jena, 2004, preprint.

[25] A. Jonsson, Atomic decomposition of Besov spaces on closed sets, Function spaces, differential operators and nonlinear analysis (Friedrichroda, 1992), 1993, pp. 285-289.

[26] _ B B B _ Bnian motion on fractals and function spaces, Math. Z. 222 (1996), no. 3, $495-504$.

[27] A. Jonsson and H. Wallin, Function spaces on subsets of $\mathbf{R}^{n}$, Math. Rep. 2 (1984), no. 1.

[28] Jun Kigami, Analysis on fractals, Cambridge Tracts in Mathematics, vol. 143, Cambridge University Press, Cambridge, 2001.

[29] T. Kumagai, Function spaces and stochastic processes on fractals, Fractal geometry and stochastics III, 2004, pp. 221-234.

[30] Y. Liu, G. Lu, and R. L. Wheeden, Some equivalent definitions of high order Sobolev spaces on stratified groups and generalizations to metric spaces, Math. Ann. 323 (2002), no. 1, 157-174. 
[31] R. A. Macías and C. Segovia, Lipschitz functions on spaces of homogeneous type, Adv. in Math. 33 (1979), no. 3, 257-270.

[32] P. Malliavin, Integration and probability, Graduate Texts in Mathematics, vol. 157, SpringerVerlag, New York, 1995.

[33] P. Mattila, Geometry of sets and measures in Euclidean spaces, Cambridge Studies in Advanced Mathematics, vol. 44, Cambridge University Press, Cambridge, 1995.

[34] V. G. Maz'ja, Sobolev spaces, Springer Series in Soviet Mathematics, Springer-Verlag, Berlin, 1985.

[35] Y. Meyer, Wavelets and operators, Cambridge Studies in Advanced Mathematics, vol. 37, Cambridge University Press, Cambridge, 1992.

[36] S. Semmes, Some novel types of fractal geometry, Oxford Mathematical Monographs, The Clarendon Press Oxford University Press, New York, 2001.

[37] R. S. Strichartz, Function spaces on fractals, J. Funct. Anal. 198 (2003), no. 1, 43-83.

[38] H. Triebel, Interpolation theory, function spaces, differential operators, North-Holland Mathematical Library, vol. 18, North-Holland Publishing Co., Amsterdam, 1978.

[39] _ Theory of function spaces, Monographs in Mathematics, vol. 78, Birkhäuser Verlag, Basel, 1983.

[40] Theory of function spaces. II, Monographs in Mathematics, vol. 84, Birkhäuser Verlag, Basel, 1992.

[41] _ Fractals and spectra, Monographs in Mathematics, vol. 91, Birkhäuser Verlag, Basel, 1997.

[42] _ The structure of functions, Monographs in Mathematics, vol. 97, Birkhäuser Verlag, Basel, 2001.

[43] , Non-smooth atoms and pointwise multipliers in function spaces, Ann. Mat. Pura Appl. (4) 182 (2003), no. 4, 457-486.

[44] _ A note on wavelet bases in function spaces, Orlicz Centenary Vol., 2004, pp. $193-206$.

[45] - Wavelet basis in anisotropic function spaces, Proc. Conf. 'Function Spaces, Differential Operators and Nonlinear Analysis 2004', Milovy, Czech Republic, to appear.

[46] H. Triebel and D. Yang, Spectral theory of Riesz potentials on quasi-metric spaces, Math. Nachr. 238 (2002), 160-184.

[47] H. Wallin, The trace to the boundary of Sobolev spaces on a snowflake, Manuscripta Math. 73 (1991), no. 2, 117-125.

[48] P. Wojtaszczyk, A mathematical introduction to wavelets, London Mathematical Society Student Texts, vol. 37, Cambridge University Press, Cambridge, 1997.

[49] D. Yang, Frame characterizations of Besov and Triebel-Lizorkin spaces on spaces of homogeneous type and their applications, Georgian Math. J. 9 (2002), no. 3, 567-590.

[50] _ Besov spaces on spaces of homogeneous type and fractals, Studia Math. 156 (2003), no. $1,15-30$.

[51] _ Some function spaces relative to Morrey-Campanato spaces, Nagoya Math. J., to appear.

[52] M. Zähle, Harmonic calculus on fractals - a measure geometric approach II, Trans. Amer. Math. Soc., to appear.

[53] Riesz potentials and Liouville operators on fractals, Potential Anal. 21 (2004), no. 2, 193-208. 